\title{
¿The Interannual Variability of the Tropical Divergence Tilt and Its Connection with the Extratropical Circulation
}

\author{
PABLO ZURITA-GOTOR \\ Instituto de Geociencia, Universidad Complutense Madrid-Consejo Superior de Investigaciones Científicas, Madrid, Spain
}

(Manuscript received 20 May 2020, in final form 6 August 2020)

\begin{abstract}
Previous theoretical work has suggested that the strength of the divergent eddy momentum flux in the deep tropics, due to correlations between rotational zonal velocities and divergent meridional velocities, increases with the meridional tilt of the large-scale divergence field. To test that idea, this work investigates the interannual variability of the divergent eddy momentum flux in reanalysis data. Consistent with the theory, it is found that the eddy momentum flux variability is driven by two main parameters: the amplitude of the tropical stationary wave and the tilt of the divergence field. Together, these two parameters account for $80 \%(90 \%)$ of the interannual eddy momentum flux variance during boreal (austral) winter. The interannual variability of these parameters is governed by the internal atmospheric dynamics. During boreal winter, interannual changes in MJO variability explain nearly half of the interannual variance in the stationary wave amplitude, depending on whether on average MJO anomalies interfere constructively or destructively with the stationary wave. The interannual variability of the divergence phase tilt is modulated by tropical-extratropical interactions in the Pacific. The tilt increases during the negative phase of the west Pacific Oscillation associated with a dipole of upper-level divergence (convergence) on the northern (southern) side of the Pacific jet exit region.
\end{abstract}

KEYWORDS: Ageostrophic circulations; Atmosphere-ocean interaction; Planetary waves; Teleconnections; Interannual variability; North Pacific Oscillation

\section{Introduction}

One of the most distinctive features of the precipitation field in the tropics is the slanted structure of the southern branch of the ITCZ along the South Pacific convergence zone (SPCZ; Trenberth 1976). Similar but weaker tilted convergence zones also exist over the two other tropical Southern Ocean basins, and much research is being done to understand these tilted structures (e.g., Widlansky et al. 2011; van der Wiel et al. 2015, 2016).

Although no similar tilted structure has been identified in the Northern Hemisphere, we have recently noted (ZuritaGotor 2019b, 2020) that the large-scale divergence field also tilts eastward moving away from the equator in this hemisphere, even if this may not be obvious in the full precipitation field due to the impact of the fine ITCZ structure. The tilting of the divergence field only becomes apparent when this field is filtered to highlight the large scales-see, for example, the climatological velocity potential in Fig. 1.

The simplest explanation for the tilted structure is that it is forced by the boundary conditions, either the continental distribution or the underlying SST. However, modeling studies of the SPCZ suggest that this phenomenon has a dynamical origin (van der Wiel et al. 2016). Additionally, aquaplanet models with zonally varying but untilted SST anomalies are found to produce tilted precipitation fields (see, e.g., Fig. 4 of Ting and Held 1990). In fact, even the simple Gill (1980) model

¿ Denotes content that is immediately available upon publication as open access.

Corresponding author: Pablo Zurita-Gotor, pzurita@alum.mit.edu produces a tilted divergence field, though this again may only be obvious when forced with large-scale heating as in Showman and Polvani (2011). A number of different mechanisms could plausibly make the divergence tilt, involving the background horizontal shear, tropical-extratropical interactions, moist dynamics, etc. The dominant mechanism may not be the same in all models. For instance, Zurita-Gotor (2020) attributed the Gill-model tilt to the impact of Newtonian cooling, which we would not expect to play a dominant role in the terrestrial atmosphere.

On the other hand, Zurita-Gotor (2019b, 2020) have noted that the divergence tilt determines the direction of the dominant contribution to the eddy momentum flux in the deep tropics, which is due to correlations between rotational (nondivergent) zonal wind anomalies and divergent (irrotational) meridional wind anomalies $\overline{u_{r}^{\prime} v_{d}^{\prime}}$. Zurita-Gotor (2019a) refers to this term as the divergent eddy momentum flux. In particular, for the inviscid Gill problem Zurita-Gotor (2020) shows that the domain-integrated divergent eddy momentum flux by zonal wavenumber $k$ is given by

$$
\left\langle\overline{u_{r}^{\prime} v_{d}^{\prime}}(k)\right\rangle=\int_{-\infty}^{\infty} 2 \frac{l}{k} \hat{K}_{d}(k, l) d l,
$$

where angle brackets stand for meridional integrals, overbars and primes denote zonal-mean and eddy anomalies, respectively, and $r$ and $d$ subscripts rotational and divergent components, respectively. The term $\hat{K}_{d}(k, l)$ is the spectrum of divergent eddy kinetic energy, where $k$ and $l$ are the zonal and meridional wavenumbers. The above relation implies that the sign of the integrated momentum flux depends on the mean tilt of the divergence field, as weighted by $\hat{K}_{d}(k, l)$. 

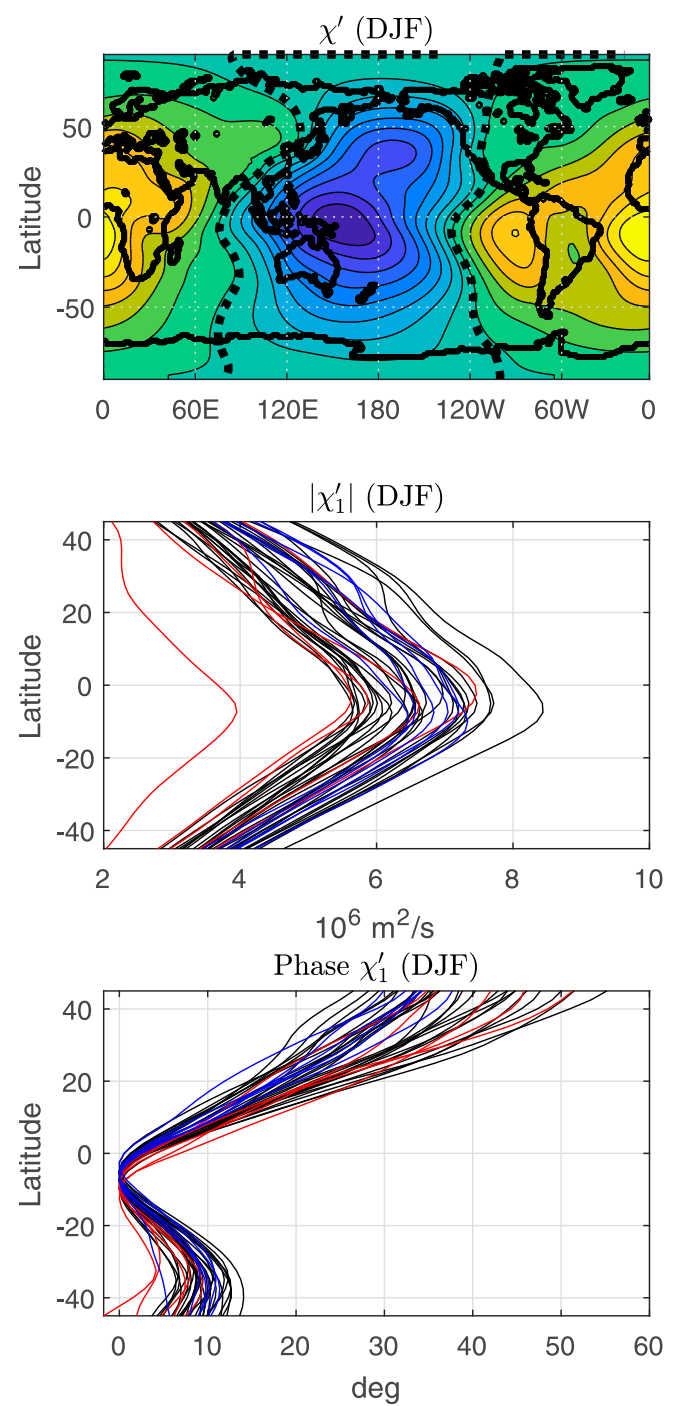

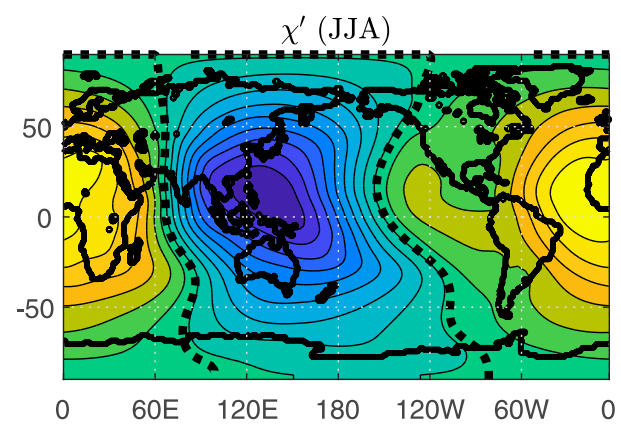

$\left|\chi_{1}^{\prime}\right|(\mathrm{JJA})$

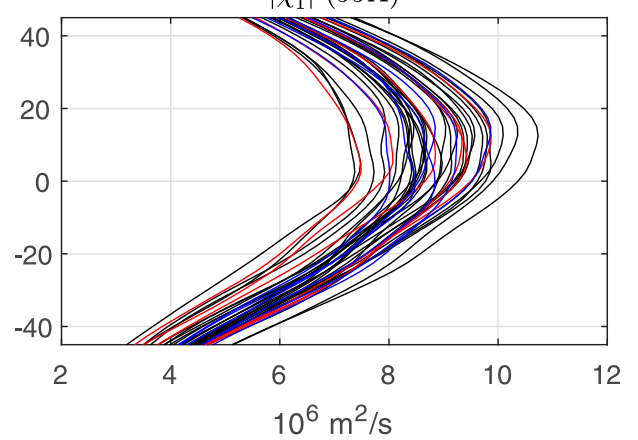

Phase $\chi_{1}^{\prime}(\mathrm{JJA})$

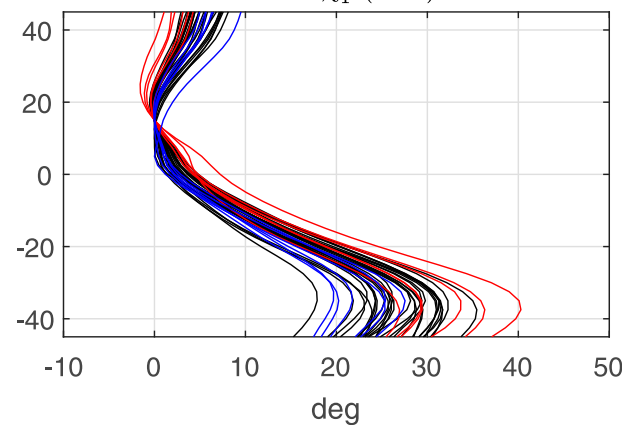

FIG. 1. (top) Climatological mean velocity potential over the 100-350-hPa layer during (left) DJF and (right) JJA. (middle) Amplitude of the $k=1$ harmonic of the velocity potencial for individual DJF and JJA seasons. Years with warm (cold) ENSO phases are shown with red (blue) lines. (bottom) As in the middle panels, but for the phase of the $k=1$ harmonic.

The relation between divergence tilt and eddy momentum flux led Zurita-Gotor (2019b) to speculate that the observed tilt might be a signature of meridional propagation. ZuritaGotor (2020) provided some evidence supporting this claim in a simple shallow-water model. Kraucunas and Hartmann (2007) had previously shown that in the presence of a Hadley cell, the eddy momentum flux in that model is directed against the Hadley flow-consistent with along-stream propagation — and increases with Hadley cell strength. Zurita-Gotor (2020) showed that the eddy momentum flux increase with Hadley cell strength in this model is accompanied by an increased divergence tilt in the downstream Hadley cell direction and is well captured by Eq. (1) when the ensuing changes in the $\hat{K}_{d}(k, l)$ spectrum are taken into account.

The goal of this paper is to test some of these theoretical ideas using the observed interannual variability. The main questions that we aim to address are the following:
- What determines the interannual variability of the divergent eddy momentum flux in the deep tropics? Are changes in the divergence tilt important for that variability?

- What processes are responsible for the interannual variability of the divergence tilt? Is meridional propagation affected by changes in the divergence tilt? Is there an extratropical impact?

This paper is organized as follows. Section 2 describes the data and introduces two parameters to characterize the amplitude and tilt of the large-scale tropical divergence. Section 3 investigates the relation between interannual changes in these two parameters and in the tropical eddy momentum flux. The remaining two sections focus on the variability of these parameters: section 4 seeks for tropical drivers while section 5 investigates the connection to the extratropical circulation. We conclude with a summary and some discussion in section 6 . 


\section{Data and methods}

Zurita-Gotor (2019b, 2020) discusses the relation between the meridional tilt of the coarse-grained divergence field and the tropical eddy momentum fluxes in the tropics. In both studies, a simple Fourier filter is used to retain only the gravest meridional harmonics of the divergence field. In this work, we will instead characterize the large-scale divergence using the velocity potential: $\chi=\nabla^{-2} D$, with no need for filtering.

The top panels of Fig. 1 show the climatological uppertroposphere (100-350-hPa average) $\chi$ for the two solstice seasons, computed using ERA-Interim data (Dee et al. 2011) over its full period of availability (January 1979-August 2019). The velocity potential is dominated by zonal wavenumber 1 and displays planetary meridional structure. Most relevant for the present study, $\chi$ tilts eastward with latitude moving away from its maximum near the equator in the summer hemisphere. Although this tilt is observed in both hemispheres and seasons, it is more pronounced in the winter hemisphere.

We will focus on the interannual variability of this structure, which we characterize using the dominant zonal harmonic $k=1$. Zurita-Gotor (2019a) shows that this harmonic also provides the leading contribution to the tropical eddy momentum flux. Focusing on a single harmonic allows us to unambiguously characterize the tilt of the velocity potential using a simple Fourier transform, even if its variability does not solely occur at this scale. For instance, the meridional tilts for zonal waves $k=1$ and $k=2$ are correlated at a $95 \%$ significance level during DJF and at a $99 \%$ significance level during JJA in interannual time scales (details on the computation of these tilts are provided below).

The bottom two rows of Fig. 1 show the amplitude and phase of the $\hat{\chi}_{1}$ harmonic for all ERA-Interim years. For later reference, warm (cold) ENSO years are emphasized using red (blue) lines. All phases are defined relative to their minimum value in the deep tropics, found near the latitude of maximum wave amplitude in the summer hemisphere.

There is significant interannual variability in both the amplitude and phase of $\hat{\chi}_{1}$. We introduce two parameters to characterize this variability: the peak velocity potential amplitude $\chi_{1}$ and the mean velocity potential tilt over the winter hemisphere $\ell$. The latter is defined as the net phase difference in degrees of the $\hat{\chi}_{1}(y)$ field between a reference latitude $\phi_{0}$ and a latitude $25^{\circ}$ apart moving into the winter hemisphere (results are not sensitive to the precise separation). The reference latitude $\phi_{0}$ is taken to be the latitude of minimum phase for the climatological $\hat{\chi}_{1}$ field $\left(7.5^{\circ} \mathrm{S}\right.$ during DJF and $12.5^{\circ} \mathrm{N}$ during JJA). We have chosen to keep this reference latitude constant instead of using the latitude of minimum phase for each year because this minimum is not always well defined, which adds considerable noise to the interannual $\ell$ variability, especially during JJA. The results presented in this paper are robust if a varying $\phi_{0}$ is used during DJF, though correlations are degraded. These two parameters, $\chi_{1}$ and $\ell$, are not significantly correlated with each other.

We investigate the relation between changes in these two parameters and in the atmospheric circulation in interannual time scales. The circulation is characterized using ERAInterim data at $2.5^{\circ}$ resolution, averaged for each of the available 40 (41) DJF (JJA) seasons. Stationary eddy fluxes are computed for each of these seasons using seasonal-mean eddy fields (eddy components are defined as differences from the zonal-mean) and as such vary interannually. We also investigate the interannual variability of the extratropical storm track, defined in terms of seasonal-mean bandpass-filtered (2-7-days Lanczos filter) transient eddy fluxes.

We connect our results with known modes of climate variability, depicted using standard indices. ENSO is assessed using the oceanic Niño index (mean SST over the Niño-3.4 region, $120^{\circ} \mathrm{E}-170^{\circ} \mathrm{W}, 5^{\circ} \mathrm{S}-5^{\circ} \mathrm{N}$ ) computed using NOAA ERSST data (Huang et al. 2015). An index for the west Pacific Oscillation (Wallace and Gutzler 1981) is computed subtracting the average $500-\mathrm{hPa}$ height over the regions $25^{\circ}-40^{\circ} \mathrm{N}$, $140^{\circ} \mathrm{E}-150^{\circ} \mathrm{W}$ and $50^{\circ}-70^{\circ} \mathrm{N}, 140^{\circ} \mathrm{E}-150^{\circ} \mathrm{W}$ (https://psl.noaa. gov/data/timeseries/daily/WPO/). Finally, we use the real-time multivariate MJO (RMM) method (Wheeler and Hendon 2004) to estimate the daily amplitude and phase of the Madden-Julian oscillation (data downloaded from http:// www.bom.gov.au/climate/mjo).

For the regression analyses, significance is estimated using a two-tailed Student $t$ test, taking each year to be independent (the interannual autocorrelation of $\ell$ and $\chi_{1}$ is weak). We use the following graphical conventions to depict significance across all plots. In line plots, 95\% (99\%) significant values are indicated with empty (filled) markers. In color shading plots, these significance levels are indicated with black/red stippling.

In some analyses we distinguish between warm, cold, or neutral ENSO years. This categorization is based on the standarized ENSO index over the period of study, with warm/ cold ENSO events exceeding a positive/negative standard deviation.

\section{Drivers of tropical eddy momentum flux variability}

As discussed in the introduction, our previous theoretical work suggests that the divergent eddy momentum flux should increase with $\ell$ in the tropics. In this section, we test that idea by examining the relation between interannual changes in $\ell$ and in the tropical eddy momentum flux. As in our previous work, we focus on the stationary $\overline{u_{r}^{\prime} v_{d}^{\prime}}$ due to zonal wave $k=1$. ZuritaGotor (2019a) shows that the contribution by this harmonic represents about $70 \%(90 \%)$ of the total divergent momentum flux during DJF (JJA).

Figure 2 (top) shows with blue line the regression of this momentum flux integrated over the 350-100-hPa layer. Consistent with the theoretical analysis of Zurita-Gotor (2020), the southward (northward) cross-equatorial eddy momentum flux during DJF (JJA) increases with meridional tilt, though the DJF regression fails to reach the $95 \%$ significance level. Reversibly, the bottom panels of Fig. 2 show the regression of the $\widehat{\chi_{1}}$ phase on the upper-troposphere $\overline{u_{r}^{\prime} v_{d}^{\prime}}$, integrated from $10^{\circ} \mathrm{S}$ to $10^{\circ} \mathrm{N}$. From this perspective, increases in the tropical eddy momentum flux are associated with an enhanced divergence tilt at the $99 \%$ significance level.

Of course, the eddy momentum flux should depend not only on $\ell$ but also on the strength of the stationary wave. To assess their relative importance, Fig. 3 shows scatterplots of the mean tropical $\overline{u_{r}^{\prime} v_{d}^{\prime}}$ for the individual years against $\ell$ (Fig. 3, left) and 

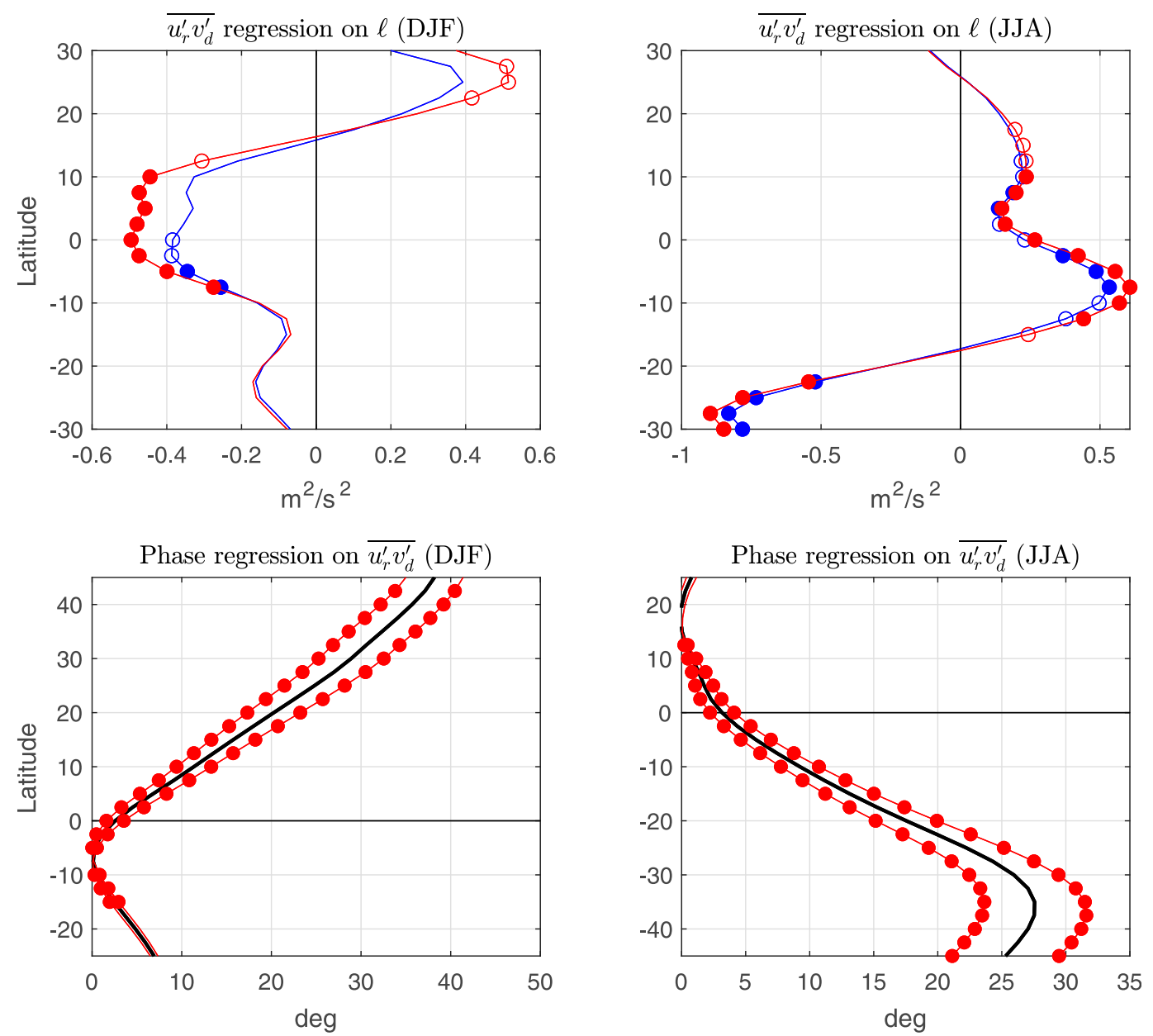

FIG. 2. (top) In blue, regression of the seasonal-mean upper-troposphere (100-350-hPa mean) divergent momentum flux on the divergence tilt $\ell$ during (left) DJF and (right) JJA. The red curves show the same after subtracting the $\overline{u_{r}^{\prime} v_{d}^{\prime}}$ variability linearly congruent with $\chi_{1}$ (see text for details). Empty (filled) markers are used to indicate $95 \%$ (99\%) significance based on a two-tailed $t$ test. (bottom) Climatological mean phase of the velocity potential $k=1$ harmonic (black), with its regression on the tropical-mean $\left(10^{\circ} \mathrm{S}-10^{\circ} \mathrm{N}, 100-350 \mathrm{hPa}\right) \overline{u_{r}^{\prime} v_{d}^{\prime}}$ added/ subtracted (red lines, significance as above).

$\chi_{1}$ (Fig. 3, center). Although all correlations are positive and significant above the 99\% level $[c=0.46(0.53)$ for $\ell$ and $c=$ 0.67 (0.73) for $\chi_{1}$ during DJF (JJA)], the large scatter implies that each of these parameters in isolation explains a limited amount of variance. Since $\ell$ and $\chi_{1}$ are uncorrelated, they both represent independent sources of $\overline{u_{r}^{\prime} v_{d}^{\prime}}$ variability.

A multiple linear regression model incorporating both parameters performs much better and explains as much as $80 \%$ (90\%) of the $\overline{u_{r}^{\prime} v_{d}^{\prime}}$ variance during DJF (JJA). To illustrate the improved fit, the right column of Fig. 3 shows a scatterplot between $\overline{u_{r}^{\prime} v_{d}^{\prime}}$ and $\ell$ after subtracting from the former the variability linearly congruent with $\chi_{1}[c=0.78(0.87)$ during DJF (JJA), $p<$ 0.001 in both cases]. The "noise" introduced by the $\chi_{1}$-driven variability explains the weak significance of the $\overline{u_{r}^{\prime} v_{d}^{\prime}}$ regression on $\ell$ during DJF noted above. When this variability is removed the regression increases slightly, but the significance now exceeds $99 \%$ over the whole deep tropics (red line in Fig. 2).
After demonstrating the relevance of $\ell$ and $\chi_{1}$ for the tropical eddy momentum flux, we investigate in the next sections how changes in these two parameters relate to the tropical and extratropical circulation.

\section{Relation with the tropical circulation}

To provide a broad overview on the scales of $\ell$ variability, Fig. 4 (top) shows its time series over the reanalysis period. There is a clear correlation with ENSO (warm/cold ENSO years are indicated with markers), also apparent in Fig. 1 (DJF correlation: $0.33, p=0.04$; JJA correlation: $0.72, p<0.001$ ). Additionally, a downward $\ell$ trend is found during both DJF and JJA.

To make sure that this trend is not bogus, we have repeated the analyses using the JRA-55 reanalysis (Kobayashi et al. 2015) over its longer availability period (from January 1958 to the present). The results are also shown in Fig. 4. There is good 

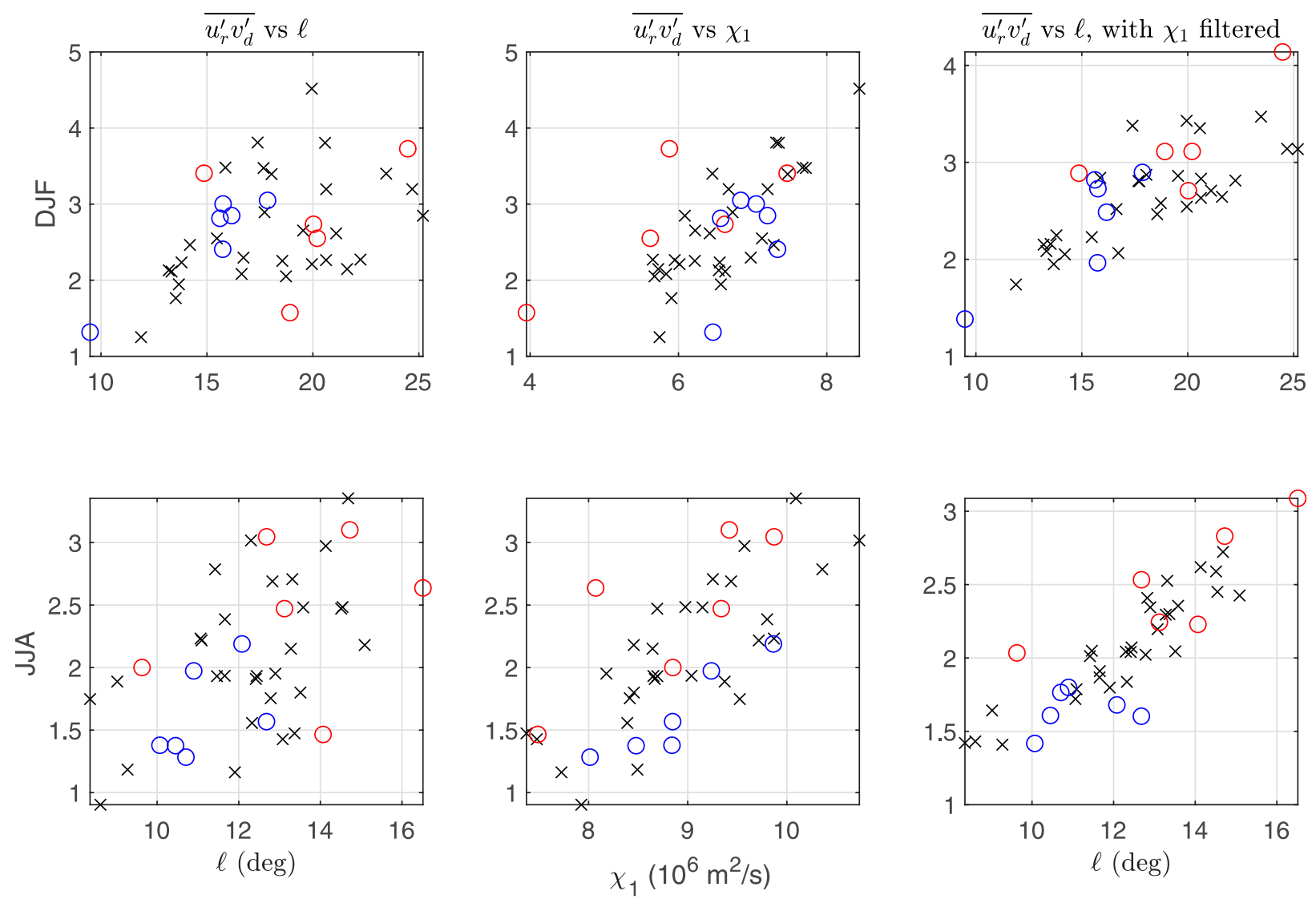

FIG. 3. (left) Scatterplot between the tropical-mean $\left(10^{\circ} \mathrm{S}-10^{\circ} \mathrm{N}, 100-350 \mathrm{hPa}\right) \overline{u_{r}^{\prime} v_{d}^{\prime}}$ and the divergence phase tilt $\ell$ for all (top) DJF or (bottom) JJA seasons. Seasons with warm (cold) ENSO phases are emphasized with red (blue) markers. (center) As in the left panels, but against the stationary wave amplitude $\chi_{1}$. (right) As in the left panels, but against the phase tilt with the $\overline{u_{r}^{\prime} v_{d}^{\prime}}$ variability linearly congruent with $\chi_{1}$ substracted out.

but imperfect agreement between the two estimates, which is not surprising because $\ell$ is a processed product of a poorly observed field. The downward $\ell$ trend is also found for JRA-55 during the common postsatellite period but disappears during the early years. This behavior is consistent with interdecadal variability of $\ell$. A possible driver for this variability is the interdecadal Pacific oscillation (IPO), which exhibits a similar downward trend over the recent period [the middle-left panels of Fig. 4 show the IPO tripole index of Henley et al. (2015)].

Although we focus on ERA-Interim in this paper, we have checked that the results are similar for JRA-55. As a difference, the correlation with ENSO is significantly smaller for JRA-55 (0.16 during DJF and 0.33 during JJA, only the latter is $95 \%$ significant). However, this is due to differences in the period of study rather than to differences in the contemporaneous data. As shown in Fig. 4 (bottom), the relation between $\ell$ and ENSO is nonstationary: they are positively correlated after the climate transition around 1980 but not before. Changes in the atmospheric circulation around this date are well documented, particularly over the Pacific sector and in connection with ENSO (e.g., He et al. 2013). As regards $\chi_{1}$, a negative correlation with ENSO is observed during DJF but not during JJA. However, the correlation is not robust through the full period and is likely inflated by a single event (the strong 1997/ 98 El Niño, a prominent outlier in Fig. 1).

Seeking for other oceanic drivers of $\ell$ and $\chi_{1}$ variability, we have regressed the SST on these parameters considering only years with weak ENSO (standarized ENSO index below \pm 1 ). For $\chi_{1}$, no robust signal was found in any season (not shown). For $\ell$, SST anomalies are weak and mostly extratropical, and appear to be driven by the atmosphere (cf. Fig. 7). These will be discussed in more detail in section 5 .

This suggests that except for the nonstationary ENSO impact described above, changes in $\ell$ and $\chi_{1}$ are mainly due to the internal atmospheric variability. In the tropics, the MJO is the most important mode of variability at intraseasonal time scales. Grise and Thompson (2012) and Zurita-Gotor (2019a) have noted that the tropical eddy momentum fluxes undergo a substantial modulation through the MJO cycle depending on whether the MJO anomaly intereferes constructively or destructively with the stationary wave. Given the slow MJO evolution, a substantial amount of interannual tropical variability may result from MJO sampling (Hendon et al. 1999).

We have investigated the relation between interannual changes in $\ell$ and $\chi_{1}$ and the internal MJO variability, as depicted by the diagnostics of Wheeler and Hendon (2004). For 

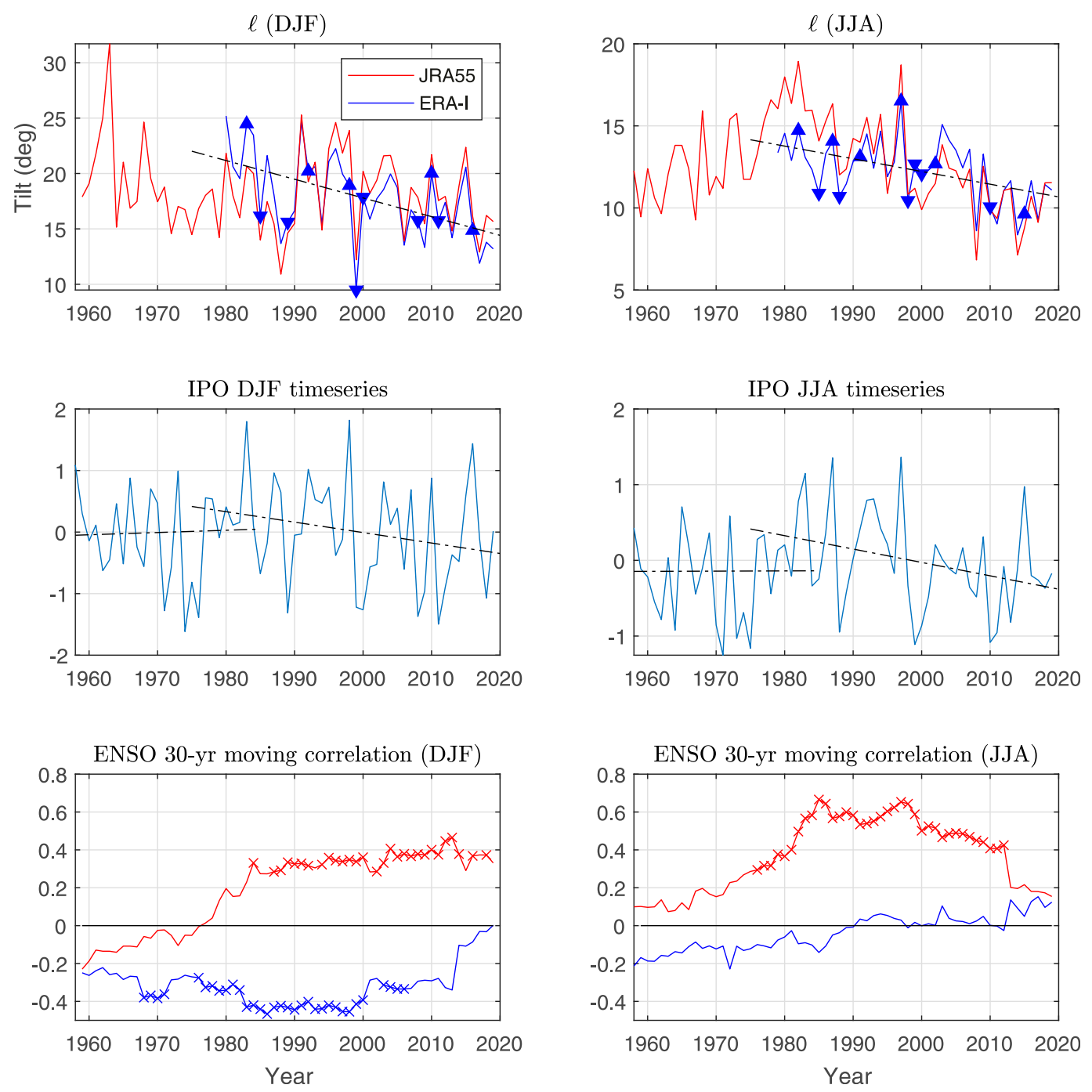

FIG. 4. (top) Time series of the divergence tilt $\ell$ for ERA-Interim (blue) and JRA-55 (red) during (left) DJF and (right) JJA. The black dash-dotted lines show linear fits to the ERA-Interim data and warm/cold ENSO years are indicated with upward/downward-pointing markers. (middle) Tripole index of the interdecadal Pacific oscillation, with linear fits to the pre- and post-1980 data shown with black dash-dotted lines. (bottom) 30-yr moving correlation between the oceanic Niño index and $\ell$ (red) or $\chi_{1}$ (blue) during DJF and JJA in JRA-55 data. Crosses are used to mark correlations significant at the $95 \%$ level based on 1000 random realizations of the sampled data for each 30 -yr window.

each DJF or JJA season, we calculated the seasonal amplitude of each MJO phase accumulating the MJO amplitude over all the days for which that particular phase was present. The left column of Fig. 5 shows the climatological mean amplitudes for all phases during DJF (Fig. 5, top) and JJA (Fig. 5, bottom), with typical values around 15 . Assuming that all phases are equally likely during the 90 days of the season, this corresponds to typical daily amplitudes of about 1.3.

The center and right columns of Fig. 5 show the regression of the seasonal MJO phase amplitudes on $\chi_{1}$ and $\ell$, respectively. During DJF, $\chi_{1}$ increases for seasons with enhanced MJO convection over the Maritime Continent and western Pacific (phases 4-7) and decreases for other phases. Although only the phase 6 anomaly is significant, this likely reflects the limited sample size for each of the phases. The smooth structure of the regression suggests that this is not just noise: when the phases are grouped together, both the positive $\chi_{1}$ correlation with phases $4-7$ and the negative $\chi_{1}$ correlation with phases 8,1 , and 2 are significant at the $99 \%$ significance level. While one should be wary of the statistics when incorporating a posteriori information, this grouping of the phases is consistent with the notion that $\chi_{1}$ is enhanced when the MJO anomaly interferes constructively with the stationary wave. The index $I_{\mathrm{MJO}}=\sum_{4-7} A_{\mathrm{MJO}}-\sum_{8,1,2} A_{\mathrm{MJO}}$ can explain $41 \%$ of the $\chi_{1}$ variance, suggesting that MJO sampling may be responsible for almost half of the $\chi_{1}$ variability. The results are very similar 

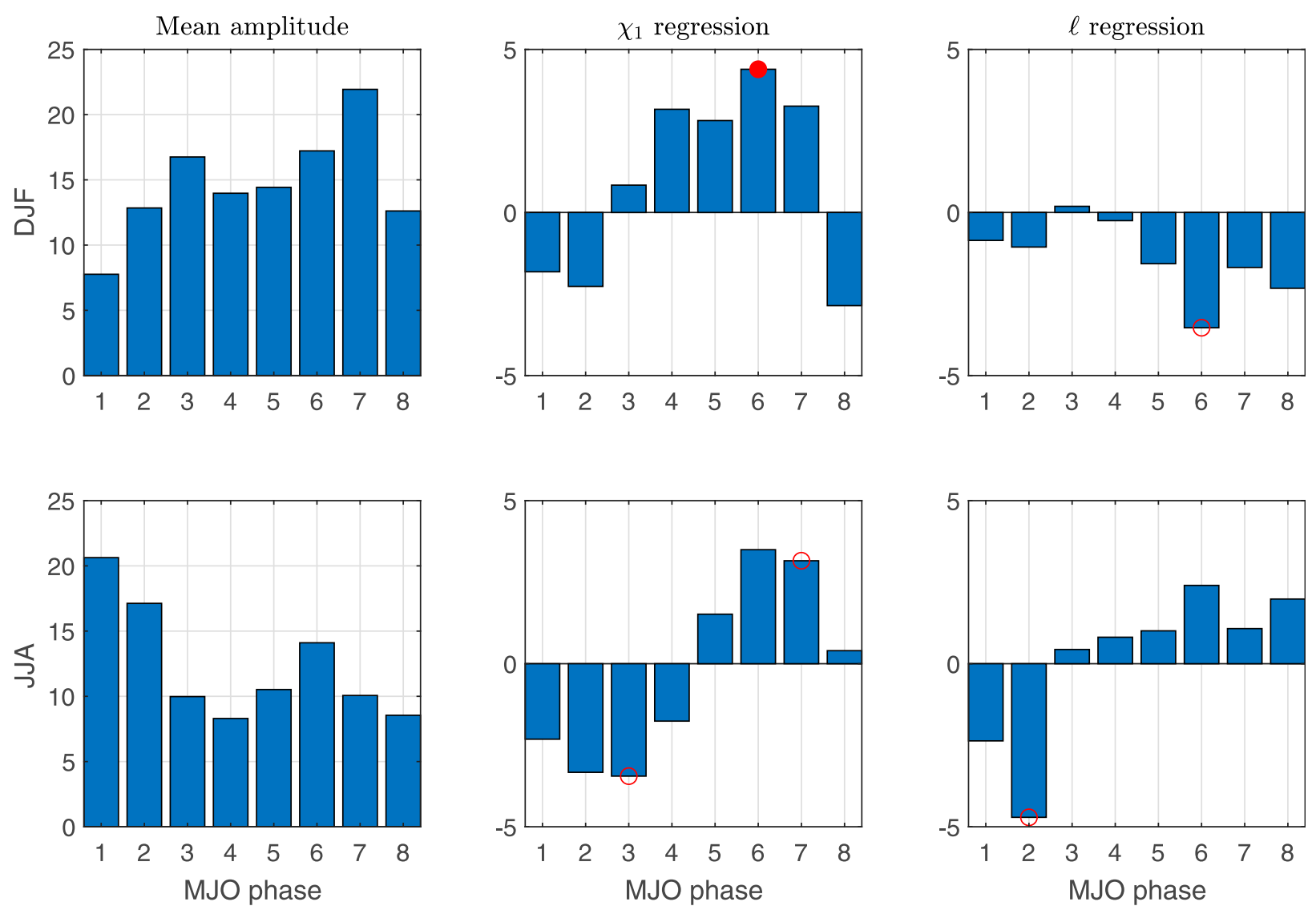

FIG. 5. (left) Climatological mean amplitude of the different MJO phases during (top) DJF and (bottom) JJA. See text for details of this calculation. (center) Regression of the phase amplitudes on $\chi_{1}$; empty (filled) red markers indicate $95 \%$ (99\%) significance. (right) As in the center panels, but for the regression on $\ell$.

when the ENSO impact on the MJO (Feng et al. 2015) is eliminated using regression analysis (not shown).

Similar results are obtained during JJA, except that the regression pattern is shifted roughly one phase eastward. However, in this case the index $I_{\mathrm{MJO}}=\sum_{5-7} A_{\mathrm{MJO}}-\sum_{1-4} A_{\mathrm{MJO}}$ only explains a $21 \%$ of the JJA interannual $\chi_{1}$ variance.

The evidence for a possible MJO influence on $\ell$ variability is less clear. The reductions in phase 6 for DJF and phase 2 for JJA appear to be significant, but only at the $95 \%$ level. Given the disparity of the results for the two seasons, and lacking a clear physical mechanism that might explain the MJO impact on $\ell$, we cannot discard that these correlations are obtained by chance. In any case, an ad hoc index constructed using a posteriori information to maximize the explained variance (adding/ subtracting the phases with positive/negative correlation) explains less than $20 \%$ of the variance during both seasons.

\section{Extratropical connection}

We showed in the previous section that the internal MJO variability can explain some of the interannual changes in $\chi_{1}$, especially during DJF, but not in $\ell$. Alternatively, the variability in these parameters might be driven from the extratropics. We have tested this idea using regression analysis. For $\chi_{1}$, no robust extratropical signal was found in any season. We describe below the anomalous global circulation associated with changes in $\ell$ focusing on DJF, when the signal is largest. To prevent confusion by the extratropical ENSO response, the regressions below are performed using only years ${ }^{1}$ with standarized ENSO index below \pm 1 .

\section{a. Anomalous circulation}

Figure 6 (top left) shows the regression of the zonal-mean zonal wind. We observe strengthening and equatorward contraction of the subtropical jet, with deceleration in the midlatitudes and acceleration over the polar cap. The polar vortex strengthens, though the $95 \%$ significance level is only reached in the lower stratosphere. There is deceleration over the equatorial upper troposphere.

\footnotetext{
${ }^{1}$ Although this does not eliminate the influence of weak/ moderate ENSO events, we found very similar results when the linear ENSO response was subtracted from the data using regression analysis (not shown). Though better suited for eliminating the impact of weak ENSO events, this alternative method can introduce extraneous variability with nonstationary ENSO modulation.
} 

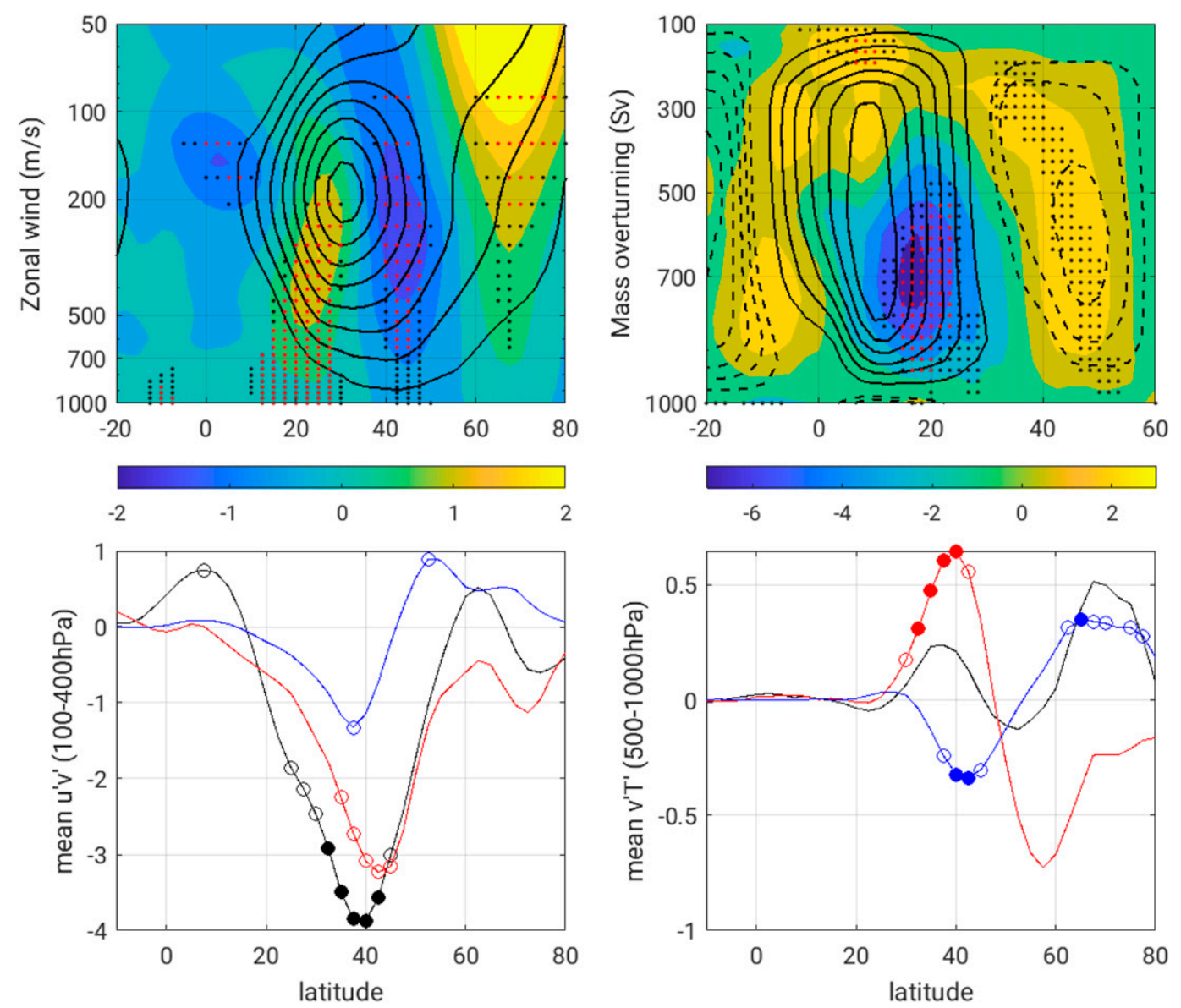

FIG. 6. (top left) Climatological DJF zonal-mean wind [contour interval (ci): $5 \mathrm{~m} \mathrm{~s}^{-1}$ ] and its regression on $\ell$ (shading). Anomalies significant at the 95\% (99\%) significance level are indicated with black (red) stippling. (top right) As in the top-left panel, but for the mass streamfunction ( $\mathrm{Sv} ; 1 \mathrm{~Sv} \equiv 10^{6} \mathrm{~m}^{3} \mathrm{~s}^{-1}$; ci: $30 \mathrm{~Sv}$ for positive contours and $10 \mathrm{~Sv}$ for negative contours). (bottom left) Regression of the meridional eddy momentum flux vertically averaged over the the 100-400 hPa layer on $\ell$ (black) and contributions by the stationary planetary eddies $(k=1-3$, red) and the synoptic transients (2-6-days bandpass filtered, blue). 95\% (99\%) significance is indicated with empty (filled) markers. (bottom right) As in the bottom-left panel, but for the meridional eddy heat flux averaged over the 500-1000-hPa layer.

The anomalous mean meridional circulation is shown in Fig. 6 (top right). The Ferrel cell weakens but there are two components to the Hadley cell response reflecting the different tropical and extratropical influences. Over the subtropical region, the Hadley cell weakens consistent with the Ferrel cell changes (Zurita-Gotor and Álvarez-Zapatero 2018). In contrast, the deep tropical Hadley cell strenghtens and expands into the summer hemisphere (implying a southward ITCZ shift), which may play some role for the equatorial deceleration noted above.

The Ferrel/Hadley changes are consistent with the regressed momentum fluxes, which show anomalous poleward Rossbywave propagation, with eddy momentum flux convergence (divergence) in the subtropics (midlatitudes). The bulk of the anomalous propagation is due to the stationary planetary eddies ( $k=1-3$, red line), with high-frequency eddies playing a small role.
Note that the negative momentum flux does not extend all the way to the equator. At this latitude, the eddy momentum flux is positive in contrast to the findings in section 3 for the divergent momentum flux (cf. to Fig. 2a). This apparent contradiction is due to the impact of the anomalous rotational momentum fluxes, which are larger than $\overline{u_{r}^{\prime} v_{d}^{\prime}}$ over this region. The implication is that the anomalous poleward propagation originates in the subtropics, where the eddy acceleration is observed, rather than in the Southern Hemisphere. The anomalous planetary eddy heat flux (Fig. 6, bottom right) suggests that this momentum flux may be partly forced by anomalous baroclinic generation.

A picture consistent with these results is the following twostep process. The anomalous convection in the Southern Hemisphere drives a zonally localized Hadley cell $v_{d}^{\prime}$, which produces a southward $\overline{u_{r}^{\prime} v_{d}^{\prime}}<0$ (equivalent to northward propagation) due to the correlation between the $u_{r}^{\prime}$ and $v_{d}^{\prime}$ 

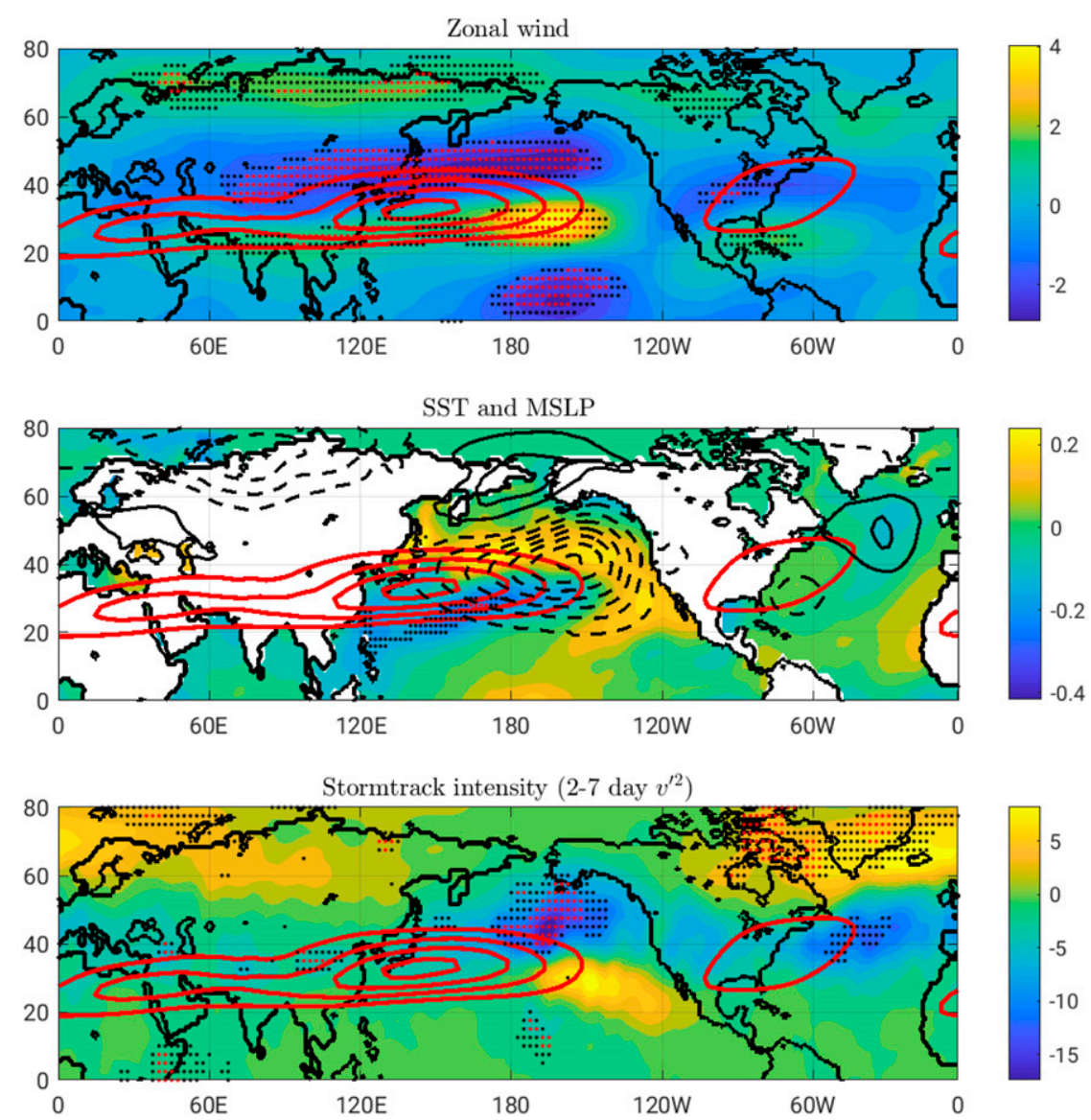

FIG. 7. (top) Regression of the upper-troposphere (100-350-hPa average) zonal wind on the divergence tilt during DJF. Anomalies significant at the 95\% (99\%) significance level are indicated with black (red) stippling. (middle) As in the top panel, but for the SST, with the regressed mean sea level pressure shown in black contours (ci: $0.5 \mathrm{hPa}$ ). (bottom) As in the top panel, but for the 2-6-day bandpass-filtered meridional velocity variance. In all panels, the red contours show the climatological jet stream (contours every $10 \mathrm{~m} \mathrm{~s}^{-1}$ starting at $30 \mathrm{~m} \mathrm{~s}^{-1}$ ).

anomalies (see Zurita-Gotor 2019a). The anomalous divergent flow and the accompanying subtropical subsidence also produce a vorticity source in the subtropics in the manner envisioned by Sardeshmukh and Hoskins (1988). The Rossby waves generated by this forcing propagate mainly poleward from the subtropics, but the weaker equatorward propagation is still sufficient to compensate near the equator the incident propagation from the Southern Hemisphere associated with the negative $\overline{u_{r}^{\prime} v_{d}^{\prime}}$. We discuss in the conclusion section 6 a possible baroclinic mechanism for the eddy momentum flux amplification, which could be regarded as a form of overreflection.

Focusing now on the horizontal structure, Fig. 7 (top) shows the regression of the upper-troposphere (100-350-hPa average) zonal wind on $\ell$. We can see that the bulk of the zonalmean signal described above comes from the North Pacific. Associated with an $\ell$ increase, the Asian-Pacific jet strengthens on its equatorward side, specially at the dowstream end. The anomalous zonal wind pattern displays a clear basin scale, although a weaker Atlantic signal is also observed downstream.
Except for its stronger upstream signal, this description is in very good agreement with the negative polarity of the west Pacific Oscillation (WP; Wallace and Gutzler 1981). This is confirmed by the high correlation $(c=-0.62, p<0.001)$ between the $\ell$ time series and the west Pacific index, which suggests that a large fraction of the interannual variability of $\ell$ is associated with this phenomenon. Figure 7 (middle) shows the anomalous sea level pressure field associated with the $\ell$ variability on top of the SST regression. The anomalous SST pattern displays the characteristic WP structure associated with atmospheric driving of the extratropical SST (Linkin and Nigam 2008).

Other anomalous fields are also in good agreement with the comprehensive WP description by Linkin and Nigam (2008). For instance, the bottom panel of Fig. 7 shows that the Pacific storm track, as measured by the bandpass-filtered (2-7 days) $v$ variance, weakens and shifts equatorward with the baroclinicity. Meridional shifts are also found for the eddy heat and vorticity fluxes (not shown) and are responsible for the zonal-mean synoptic eddy fluxes shown in Fig. 6. Likewise, the 

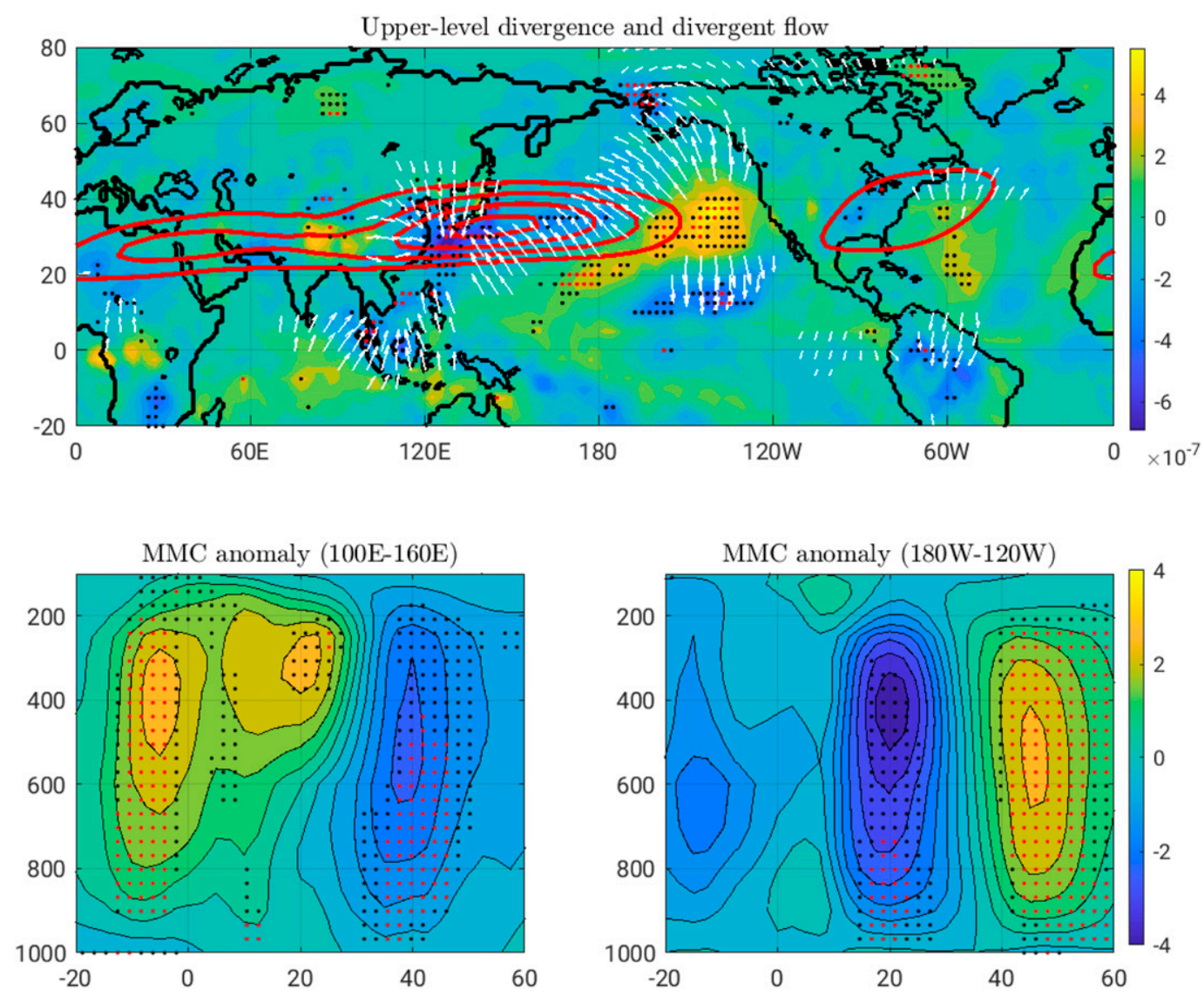

FIG. 8. (top) Regression of the upper-troposphere (100-350-hPa average) horizontal divergence (shading; significance as in Fig. 7) and the divergent wind (white vectors shown when at least one of the velocity components is significant at the $95 \%$ level) on $\ell$ during DJF. The red contours show the climatological jet stream. (bottom) Anomalous meridional overturning (Sv) over the longitude sectors (left) $100^{\circ}-160^{\circ} \mathrm{E}$ or (right) $180^{\circ}-120^{\circ} \mathrm{W}$. Significance is indicated with black/red stippling as above.

polar vortex strengthening in Fig. 6 is consistent with findings by Limpasuvan et al. (2005) that the vortex cools during the negative WP phase.

Finally, Fig. 8 describes the divergent circulation associated with the $\ell$ changes. There is anomalous divergence over the south-equatorial Indian Ocean and north of Australia and anomalous convergence north of the equator, consistent with a southward shift of convection into the summer hemisphere over this region. Farther northward in the same sector, there is strong subsidence off the coast of subtropical East Asia near the Korean Peninsula. Over the same latitudinal band, anomalous upper-level divergence is found in the east Pacific near the coast of North America, with compensating convergence to its south.

For all these centers, the anomalous divergent wind is dominated by the meridional component. To visualize this divergent meridional circulation, the bottom panels of Fig. 8 show the local Hadley circulation (Schwendike et al. 2014) averaged over the longitudinal sectors $100^{\circ}-160^{\circ} \mathrm{E}$ and $180^{\circ}-$ $120^{\circ} \mathrm{W}$. The deep cross-equatorial Hadley cell found in the zonal mean (Fig. 6) can be linked to the near-equatorial convection over the Indian-Australian sector. On the other hand, we observe a quadripolar structure centered at the subtropical/ midlatitude edge, with a direct (indirect) meridional circulation to the south (north) over the jet entrance region and the reverse over the jet exit region. This ageostrophic circulation is as required to produce the southward shift of a zonally localized jet (Martin 2013), as elaborated in detail in the next subsection.

Combined, the divergence centers described above lead to increased southwest-to-northeast tilt of the divergence field. In particular, when the equatorial convection maximum is anchored near the Maritime Continent, $\ell$ increases when the climatological convergence in the subtropical east Pacific shifts southward (implying anomalous divergence to the north), as observed during the negative WP phase.

\section{b. Dynamics of the divergent circulation}

To understand the origin of the anomalous divergent circulation, we start with the linearized perturbation vorticity equation:

$$
J\left(\psi_{\mathrm{clim}}, \delta \xi\right)+J\left(\delta \psi, f+\xi_{\mathrm{clim}}\right) \approx \delta F_{\mathrm{DIV}}+\delta F_{\mathrm{TRAN}}
$$



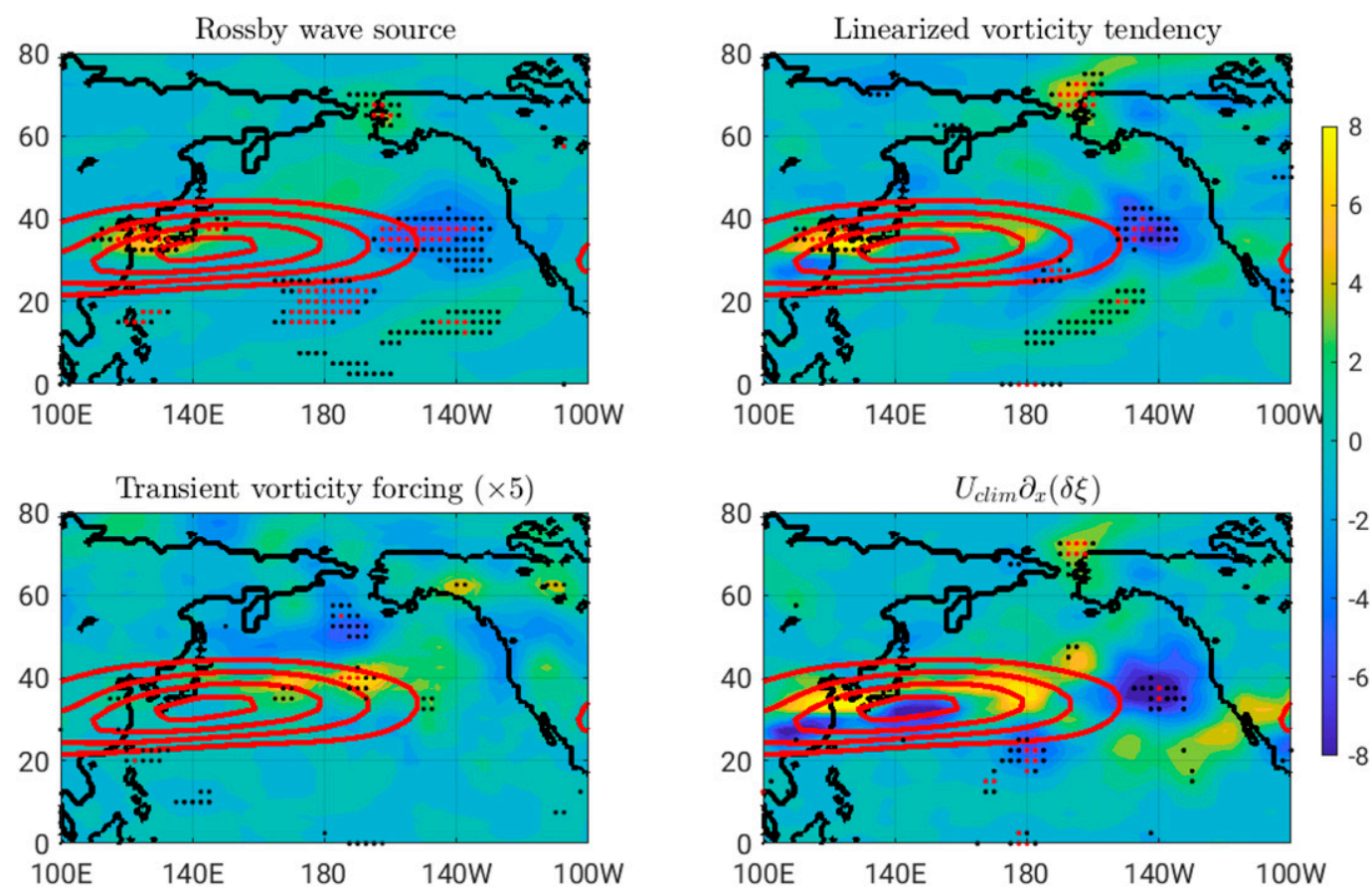

FIG. 9. (top left) Regression of the Rossby wave source $F_{\text {DIV }}$ on $\ell$ during DJF. (top right) As in the top-left panel, but for the linearized rotational vorticity tendency (see text for details). (bottom left) As in the top-right panel, but for the vorticity forcing by the high-frequency eddies, multiplied by 5. (bottom right) As in the top-right panel, but for the contribution of $U_{\operatorname{clim}} \partial_{x}(\delta \xi)$ to the rotational vorticity tendency. In all panels, the fields have been smoothed zonally using a 5-point running mean prior to computing the regression so as to focus on the large scales and eliminate the small-scale noise. Units in $10^{-11} \mathrm{~s}^{-2}$, with significance indicated with black/red stippling as in Fig. 7.

where $\xi$ is relative vorticity, $\psi$ is streamfunction, $F_{\text {TRAN }}$ is the vorticity forcing by the high-frequency transients, and $F_{\text {DIV }}=-(f+\xi) \nabla^{2} \chi-\mathbf{v}_{\chi} \cdot \nabla(f+\xi)$ is the vorticity forcing by the divergent flow (Rossby wave source) due to vortex stretching and divergent vorticity advection (Sardeshmukh and Hoskins 1988). Here and in the following, all variables represent seasonal (DJF) means unless otherwise indicated, with no special notation. The $\delta$ symbol refers to anomalous (regressed) fields, and the subscript "clim" to climatological fields.

Figure 9 shows that $\delta F_{\text {DIV }}$ agrees reasonably well with the linearized vorticity tendency on the left-hand side, while the transient eddy vorticity forcing $\delta F_{\text {TRAN }}$ is much smaller. In the deep tropics, $F_{\text {DIV }}$ may be thought of as the driving force for the vorticity dynamics because the divergent circulation is determined by the heating, independent to first order of the rotational circulation. However, as thermal advection becomes important balancing the heating in the subtropics the causality becomes ambiguous in Eq. (2). In some instances, it may be more appropriate to think of the divergent circulation and $F_{\text {DIV }}$ as determined by the rotational dynamics rather than the reverse. Additionally, as noted by Qin and Robinson (1993) tropical heating produces not only a Rossby wave source in the subtropics, but also an extratropical $F_{\text {DIV }}$ wave train that extends well beyond the tropical forcing region. This divergent circulation is best understood as a response to the temperature and vorticity tendencies produced by the rotational propagation.
In quasigeostrophic theory the ageostrophic circulation is constrained by the requirement that the vorticity and temperature are in balance: $f \partial_{p} \xi=-(R / p) \nabla^{2} T$, with $T$ governed by the thermodynamic equation:

$$
-J(\psi, T)-\left(\frac{p}{p_{0}}\right)^{R / c_{p}} \omega \theta_{p}+H_{\mathrm{TRAN}}+Q \approx 0 .
$$

Here, $H_{\text {TRAN }}$ is the heating by the high-frequency transients, $\omega \theta_{p}$ is adiabatic cooling, and $Q$ is diabatic heating. As the dynamical and dissipative sources of heat and vorticity acting in isolation in Eqs. (2) and (3) would tend to drive the atmosphere off balance, an ageostrophic/divergent circulation is required to restore it (Eliassen 1951).

The anomalous thermodynamic balance, obtained regressing each of the terms in Eq. (3) on $\ell$ (Fig. 10), shows that horizontal thermal advection balances to a good approximation adiabatic cooling, specially over the regions with strong upper-level divergence in Fig. 8. Diabatic heating (estimated as a residual in the thermodynamic equation) is noticeably weaker, and the smaller transient eddy heating peaks over the central Pacific, away from the main divergence centers. This balance suggests that the anomalous vertical motion in the subtropics is dynamically rather than diabatically forced.

The anomalous Q vectors (Hoskins et al. 1978) are consistent with this dynamical forcing as they diverge (converge) 

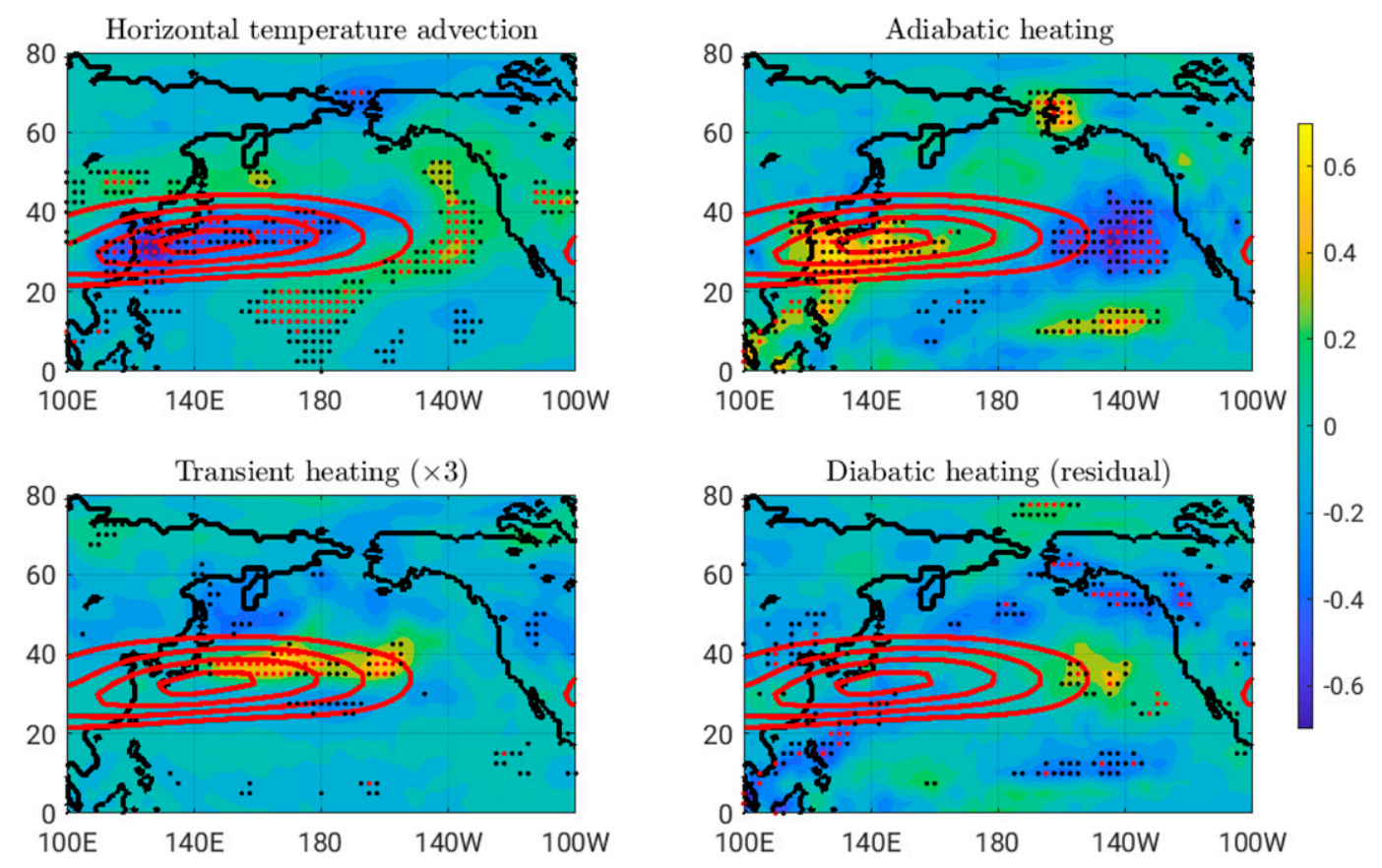

FIG. 10. (top left) Regression of the mean horizontal temperature advection ( $\mathrm{K} \mathrm{day}^{-1}$ ) over the free troposphere (300-800 hPa average) on $\ell$ during DJF. (top right) As in the top-left panel, but for the adiabatic heating. (bottom left) As in the top-right panel, but for the thermal forcing by the high-frequency eddies, multiplied by 3. (bottom right) As in the bottom-left panel, but for the diabatic heating. Significance is indicated with black/red stippling as in Fig. 7.

north of the $30^{\circ}$ latitude line near the Korean Peninsula (in the east Pacific), with compensating convergence (divergence) south of that line (Fig. 11, top). The bottom panels of this figure show the contributions of the anomalous thermal advection $-\delta\left[(R / p) \nabla^{2} J(\psi, T)\right]$ and differential vorticity advection $-\delta\left[f \partial_{p} J(\psi, f+\xi)\right]$ to the quasigeostrophic $\omega$ equation. Positive (negative) values force ascent (subsidence). At the jet entrance both thermal and vorticity advection play a role, but at the jet exit vorticity advection is by far the dominant term.

The thermal forcing at the jet entrance is the result of anomalous cold-air advection on the western side of the cyclonic anomaly, amplified by the strong land-sea thermal contrast over the region. To maintain balance, subsidence is needed to produce cyclonic (anticyclonic) forcing at upper (lower) levels. Subsidence is also induced slightly northward by upper-level anticyclonic forcing, which requires adiabatic warming for balance. Reversibly, over the jet exit region the anomalous ascent is forced by anomalous cyclonic forcing at upper levels, which requires adiabatic cooling for balance. As shown in Fig. 9 (bottom right), this cyclonic forcing is largely due to zonal advection of the upstream vorticity anomaly: $-U_{\text {clim }} \partial_{x}(\delta \xi)$.

We may thus attribute the east Pacific ascent to the zonal decay of the jet/vorticity anomaly. As shown in Fig. 12, this decay is quite sharp as we approach the American continent. To the extent that the anomalous divergence center in the east Pacific is associated with the termination of vorticity anomalies forced upstream, it can be regarded as part of the response to that forcing. To emphasize this point, Fig. 13 shows how the meridional overturning circulation in the east Pacific [as in
Schwendike et al. (2014), averaged from $120^{\circ}$ to $140^{\circ} \mathrm{W}$ and vertically integrated] is correlated with the local meridional overturning at other longitudes as a function of latitude. At all latitudes, we find a reversal of the divergent circulation between the two sides of the basin. In particular, the interannual correlation between the averaged upper-level divergence over the subtropical west $\left(110^{\circ}-150^{\circ} \mathrm{E}, 25^{\circ}-40^{\circ} \mathrm{N}\right)$ and east $\left(120^{\circ}-\right.$ $\left.160^{\circ} \mathrm{W}, 25^{\circ}-40^{\circ} \mathrm{N}\right)$ Pacific centers in Fig. 8 is $-0.7(p<0.001)$. We have also calculated the EOFs for the upper-level divergence over the North Pacific sector $\left(100^{\circ} \mathrm{E}-110^{\circ} \mathrm{W}\right.$, poleward of $\left.10^{\circ} \mathrm{N}\right)$. The leading EOF ( $21 \%$ explained variance) has a very similar structure to Fig. 8 (not shown) and its principal component is strongly correlated with the WP index $(c=0.8)$.

To conclude, we note that the vorticity anomaly in Fig. 12 also extends to latitudes with negligible vorticity forcing (cf. Fig. 9). Over these regions, the anomaly must project on the free/propagating modes of the vorticity equation. This is supported by the anomalous Plumb (1985) fluxes, which show significant meridional propagation over the central and eastern Pacific. Although the bulk of this propagation is northward, there is also some southward and cross-equatorial propagation consistent with the zonal-mean planetary eddy momentum flux in Fig. 6.

\section{c. JJA results}

When a similar regression analysis is performed during JJA, the results are much less robust and significant than during DJF. There could be several reasons for this. First, the definition of $\ell$ is more ambiguous during JJA than during DJF because the velocity potential phase minimum is not as well defined (Fig. 1), perhaps reflecting the influence of the 

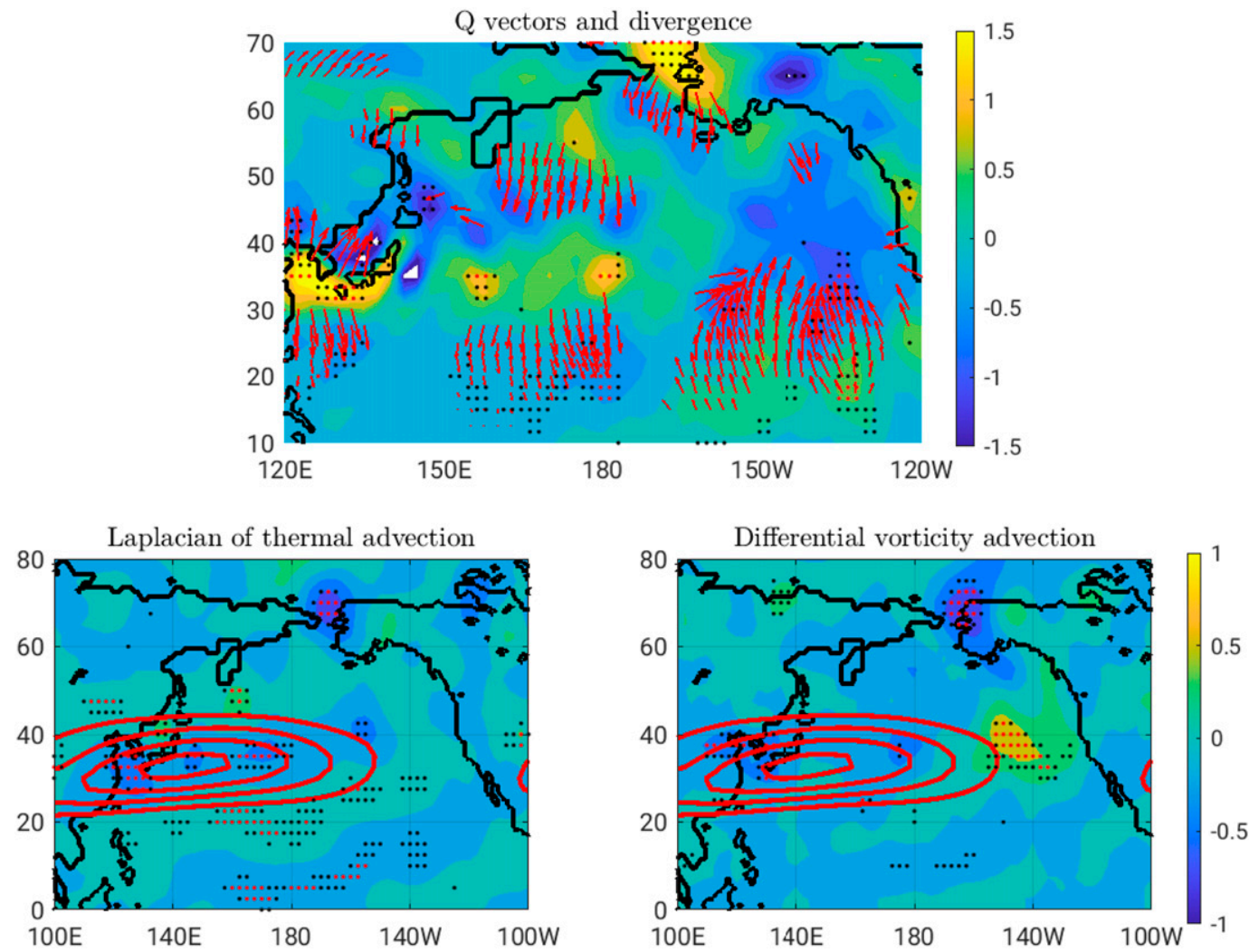

FIG. 11. (top) Regression of $\mathbf{Q}$ vectors (red vectors shown when at least one of its components is significant at the $95 \%$ level) and their divergence (shading in $10^{-19} \mathrm{~Pa}^{-1} \mathrm{~s}^{-3}$; significance as in Fig. 7) on $\ell$ during DJF, vertically averaged from 300 to $800 \mathrm{hPa}$. (bottom) Contributions of the Laplacian of (left) thermal advection $-(R / p) \nabla^{2} J(\psi, T)$ and (right) differential vorticity advection $-f \partial_{p} J(\psi, f+\xi)$ to the anomalous quasigeostrophic $\omega$ equation, vertically integrated from 300 to $800 \mathrm{hPa}$. Both forcings have been smoothed spatially using a 5-point running mean prior to computing the regression so as to focus on the large scales and eliminate the small-scale noise. Units in $10^{-14} \mathrm{~m}^{-1} \mathrm{~s}^{-3}$, with significance indicated with black/red stippling as in Fig. 7.

monsoon. Additionally, the interannual $\ell$ variability is much larger during DJF than during JJA (nearly 4 times more variance; compare the $\ell$ ranges in Fig. 3). The latter is not surprising, as the mechanism proposed to drive the $\ell$ variability during DJF relies on the termination of the vorticity anomaly over the jet exit region. The Southern Hemisphere jet is much less localized. Yet despite its smaller magnitude and weaker statistical significance, the JJA regression of upper-level divergence on $\ell$ shares some similarities with its boreal winter counterpart (Fig. 14). Near the equator, the Maritime Continent convection shifts northward into the summer hemisphere. In the subtropics, there is again anomalous east Pacific divergence at the jet exit west of the South American continent, with a hint of anomalous convergence farther west.

\section{Summary and concluding remarks}

In this study, we have used the observed interannual variability in ERA-Interim reanalysis to test the relation between divergent eddy momentum flux and meridional divergence tilt suggested by our previous work. We found that the variability of two parameters, the amplitude and meridional tilt of the $k=1$ velocity potential, can account for a large fraction ( $80 \%$ during
DJF and $90 \%$ during JJA) of the interannual eddy momentum flux variance.

Except for some nonstationary ENSO impact, these parameters are governed by the internal atmospheric dynamics. The internal MJO variability can explain some of the interannual variance in the tropical stationary wave amplitude, depending on whether it projects constructively or destructively on average on the climatological mean stationary wave. As regards the meridional divergence tilt, increases in this tilt are associated with a divergence pattern that involves (Fig. 15) (i) a meridional shift/concentration of the Maritime Continent convection into the summer hemisphere and (ii) a dipolar pattern of anomalous convergence/divergence equatorward/ poleward of the $30^{\circ}$ latitude line in the east Pacific.

While it is clear that both perturbations increase the divergence tilt, it is not obvious that they should be connected. Our regression analysis might instead be simply picking up two independent drivers of that tilt. However, we have found that the Maritime Continent convection shift is also observed for (i) a regression on the negative WP index during DJF and (ii) the leading EOF of upper-level divergence over the Pacific sector, which has a very similar structure. Additionally, we 


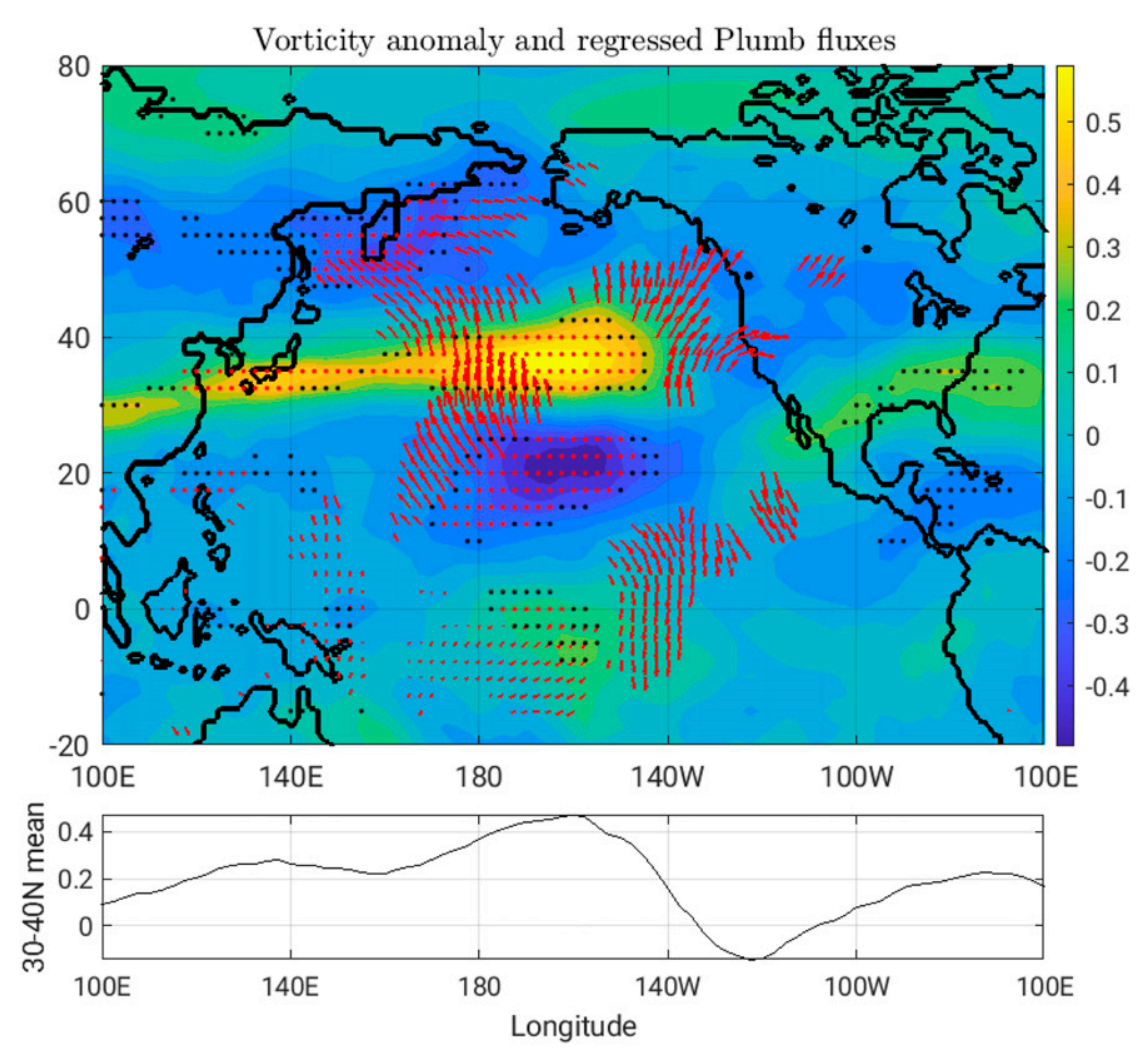

FIG. 12 (top) Regression of the upper-troposphere relative vorticity on $\ell$ (shading in days ${ }^{-1}$; significance as in Fig. 7) and anomalous Plumb vectors (red vectors shown when at least one of its components is significant at the $90 \%$ level-note that the appearance/disappearance of a vector does not necessarily imply local divergence/convergence of the flux). (bottom) Latitudinal mean of the vorticity regression over the $30^{\circ}-40^{\circ} \mathrm{N}$ latitude band.

note that the negative phase of the west Pacific Oscillation is also significantly associated with an increase in the crossequatorial divergent momentum flux during DJF.

The association between changes in the divergent crossequatorial eddy momentum flux and in extratropical variability is intriguing. Our study was motivated in part by the argument of Sardeshmukh and Hoskins (1988) that a localized Hadley cell provides a bridge for localized convection in the deep tropics to affect the extratropics by producing a Rossby wave source in the subtropics. The findings of Zurita-Gotor (2019a) that tropical eddy momentum transport occurs through a zonally localized Hadley cell provided both support and a

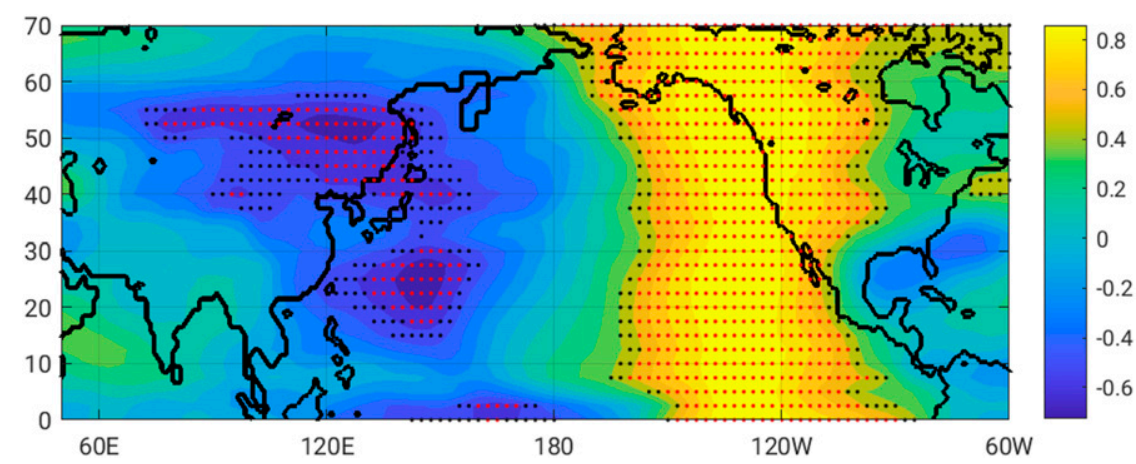

FIG. 13. Correlation between the mean meridional overturning over the east Pacific $\left(120^{\circ}\right.$ $140^{\circ} \mathrm{W}$, vertically integrated) and the local meridional overturning at other longitudes, as a function of latitude. Correlations significant at the $95 \%$ (99\%) significance level are indicated with black (red) stippling. 


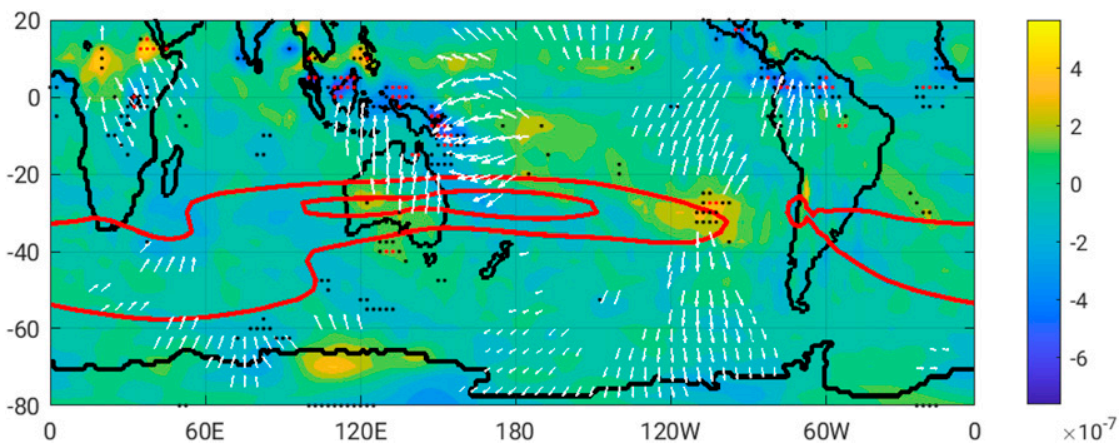

FIG. 14. Regression of the upper-troposphere (100-350-hPa average) horizontal divergence (shading; significance as in Fig. 7) and the divergent wind (white vectors shown when at least one of the velocity components is significant at the $95 \%$ level) on $\ell$ during JJA. The red contours show the climatological jet stream (contours every $10 \mathrm{~m} \mathrm{~s}^{-1}$ starting at $30 \mathrm{~m} \mathrm{~s}^{-1}$ ).

framework to test this idea quantitatively, as an eddy momentum flux is equivalent to a wave activity flux.

In principle, our results seem consistent with the arguments of Sardeshmukh and Hoskins (1988), as we found that the enhancement in the cross-equatorial eddy momentum flux is accompanied by a vorticity source in the subtropical Pacific and anomalous poleward Rossby wave propagation emanating from those latitudes. Specifically, the enhanced convection around the Maritime Continent is accompanied by compensating subsidence and cyclonic upper-level forcing near the Korean Peninsula, leading to strengthening and equatorward contraction of the subtropical jet farther downstream. The accompanying divergence dipole in the east Pacific is associated with the termination of the jet anomaly in the vicinity of the North American continent.

But as a caveat, we note that the emitted Rossby wave radiation in the subtropics is much larger than the incident divergent eddy momentum flux from the tropics, to the extent that the small fraction of backward Rossby wave radiation from the subtropics suffices to make the full cross-equatorial momentum flux poleward. Although this does not necessarily violate the pseudomomentum balance, it does require a

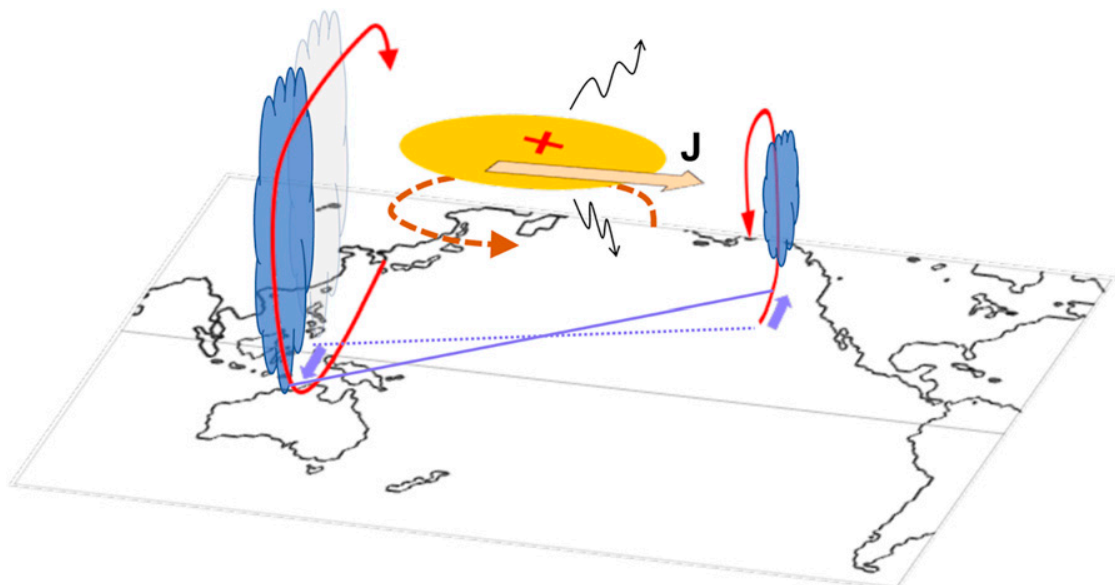

FIG. 15. Sketch illustrating the anomalous circulation associated with an increased divergence tilt during DJF. Near the Maritime Continent, convection is enhanced on the summer side of the equator and the local Hadley circulation strengthens. This produces anomalous subsidence near the Korean Peninsula and a cyclonic anomaly (orange oval) peaking farther downstream in the Pacific due to the climatological jet advection. Associated with this cyclonic anomaly, the Asian-Pacific jet strengthens on its equatorward side. The termination of the jet anomaly near the North American continent is associated with an anomalous meridional circulation in the east Pacific that produces ascent to the north and subsidence to the south. The southward (northward) shift of the divergence near the Maritime Continent (in the east Pacific) increases the divergence tilt as illustrated by the blue lines. A broken brown line shows the circulation induced by the cyclonic anomaly, which may amplify the subsidence near the Korean Peninsula through cold-air advection, and wiggly lines show the propagation of Rossby waves northward and southward from the central Pacific. 
substantial amount of overreflection and overtransmission. Overreflection and overtransmission are not possible for a zonally symmetric barotropic flow (with one-signed absolute vorticity gradient), but they can happen in a baroclinic fluid (see, e.g., Gliatto and Held 2020) and/or in an asymmetric basic state in which the stationary wave provides additional routes to amplification.

Although the classical mechanism for the instability of zonally varying flow is barotropic (Simmons et al. 1983; Swanson 2000), our analysis suggests that if overreflection does occur in this context the mechanism is likely baroclinic. While the anomalous Plumb (1985) fluxes show no hint of zonal wave accumulation (Fig. 12), there is strong anomalous baroclinic generation by the stationary planetary eddies in the subtropics (Fig. 6). Our analysis of the $\omega$ equation (Fig. 11) suggests that cold-air advection in the western Pacific is a primary forcer for the subsidence near the Korean Peninsula. This suggests a possible baroclinic mechanism for the anomaly amplification, in which a subtropical cyclonic anomaly created by tropical convection drives cold-air advection on its wake (Fig. 15), which amplifies the cyclonic anomaly through the induced vortex stretching.

However, note that the reverse causality is also possible so that the pattern could instead be driven from the extratropics, for instance by anomalous thermal advection associated with a perturbed Siberian high or Aleutian low. In that scenario, the anomalous Maritime Continent convection and cross-equatorial Hadley cell would only represent a response/feedback to the extratropical driving. In fact, such a feedback has been suggested to contribute to the strength of the East Asian winter monsoon (EAWM), so that the mean Maritime Continent pressure is also incorporated in modern EAWM indices (Wang and Chen 2014). The index of Wang and Chen is also highly correlated with the west Pacific Oscillation.

As the Asian-Pacific sector is home to a large number of climate phenomena, some of which interact in nonstationary ways, it is not possible to disentangle the drivers of the variability using observations alone. In future work, we will make use of idealized model simulations to better understand these complex interactions.

Acknowledgments. This work benefited from the comments of three anonymous reviewers. We thank the European Centre for Medium-Range Weather Forecasts, the Japan Meteorological Agency, the National Center for Atmospheric Research, and the National Oceanic and Atmospheric Administration for producing/ providing the data used in this study. The ERA-Interim data were downloaded from the ECMWF website (https://www.ecmwf.int). The JRA-55 data were obtained from the Research Data Archive at the National Center for Atmospheric Research Computational and Information Systems Laboratory (https://doi.org/10.5065/ D6HH6H41). The NOAA ERSST data were provided by the NOAA/OAR/ESRL PSD, Boulder, Colorado, from their website (http://www.esrl.noaa.gov/psd/). The author acknowledges financial support by Grant CGL2015-72259-EXP by the State Research Agency of Spain and NSF funding (Grant AGS1733818) for a summer visit to Princeton that helped motivate this study.

\section{REFERENCES}

Dee, D. P., and Coauthors, 2011: The ERA-Interim reanalysis: Configuration and performance of the data assimilation system. Quart. J. Roy. Meteor. Soc., 137, 553-597, https://doi.org/ 10.1002/qj.828.

Eliassen, A., 1951: Slow thermally or frictionally controlled meridional circulation in a circular vortex. Astrophys. Nor., 5, 19.

Feng, J., W. Chen, and X. Wang, 2015: Contrasting Madden-Julian oscillation activity during various stages of EP and CP El Niños. Atmos. Sci. Lett., 16, 32-37, https://doi.org/10.1002/ asl2.516.

Gill, A., 1980: Some simple solutions for heat-induced tropical circulation. Quart. J. Roy. Meteor. Soc., 106, 447-462, https:// doi.org/10.1002/qj.49710644905.

Gliatto, M. T., and I. M. Held, 2020: Overtransmission of Rossby waves at a lower-layer critical latitude in the two-layer model. J. Atmos. Sci., 77, 859-870, https://doi.org/10.1175/JAS-D-190055.1.

Grise, K. M., and D. W. Thompson, 2012: Equatorial planetary waves and their signature in atmospheric variability. J. Atmos. Sci., 69, 857-874, https://doi.org/10.1175/JAS-D-11-0123.1.

He, S., H. Wang, and J. Liu, 2013: Changes in the relationship between ENSO and Asia-Pacific midlatitude winter atmospheric circulation. J. Climate, 26, 3377-3393, https://doi.org/ 10.1175/JCLI-D-12-00355.1.

Hendon, H. H., C. Zhang, and J. D. Glick, 1999: Interannual variation of the Madden-Julian oscillation during austral summer. J. Climate, 12, 2538-2550, https://doi.org/10.1175/1520-0442(1999)012<2538: IVOTMJ $>2.0 . \mathrm{CO} ; 2$.

Henley, B. J., J. Gergis, D. J. Karoly, S. Power, J. Kennedy, and C. K. Folland, 2015: A tripole index for the interdecadal Pacific oscillation. Climate Dyn., 45, 3077-3090, https://doi.org/10.1007/ s00382-015-2525-1.

Hoskins, B., I. Draghici, and H. Davies, 1978: A new look at the $\omega$-equation. Quart. J. Roy. Meteor. Soc., 104, 31-38, https:// doi.org/10.1002/qj.49710443903.

Huang, B., and Coauthors, 2015: Extended Reconstructed Sea Surface Temperature version 4 (ERSST. v4). Part I: Upgrades and intercomparisons. J. Climate, 28, 911-930, https://doi.org/ 10.1175/JCLI-D-14-00006.1.

Kobayashi, S., and Coauthors, 2015: The JRA-55 reanalysis: General specifications and basic characteristics. J. Meteor. Soc. Japan, 93, 5-48, https://doi.org/10.2151/jmsj.2015-001.

Kraucunas, I., and D. L. Hartmann, 2007: Tropical stationary waves in a nonlinear shallow-water model with realistic basic states. J. Atmos. Sci., 64, 2540-2557, https://doi.org/10.1175/ JAS3920.1.

Limpasuvan, V., D. L. Hartmann, D. W. Thompson, K. Jeev, and Y. L. Yung, 2005: Stratosphere-troposphere evolution during polar vortex intensification. J. Geophys. Res., 110, D24101, https://doi.org/10.1029/2005JD006302.

Linkin, M. E., and S. Nigam, 2008: The North Pacific Oscillationwest Pacific teleconnection pattern: Mature-phase structure and winter impacts. J. Climate, 21, 1979-1997, https://doi.org/ 10.1175/2007JCLI2048.1.

Martin, J. E., 2013: Mid-Latitude Atmospheric Dynamics: A First Course. John Wiley \& Sons, 366 pp.

Plumb, R. A., 1985: On the three-dimensional propagation of stationary waves. J. Atmos. Sci., 42, 217-229, https://doi.org/10.1175/ 1520-0469(1985)042\%3C0217:OTTDPO\%3E2.0.CO;2.

Qin, J., and W. A. Robinson, 1993: On the Rossby wave source and the steady linear response to tropical forcing. J. Atmos. Sci., 50, 
1819-1823, https://doi.org/10.1175/1520-0469(1993)050<1819: OTRWSA $>2.0 . \mathrm{CO} ; 2$.

Sardeshmukh, P. D., and B. J. Hoskins, 1988: The generation of global rotational flow by steady idealized tropical divergence. J. Atmos. Sci., 45, 1228-1251, https://doi.org/10.1175/15200469(1988)045<1228:TGOGRF>2.0.CO;2.

Schwendike, J., P. Govekar, M. J. Reeder, R. Wardle, G. J. Berry, and C. Jakob, 2014: Local partitioning of the overturning circulation in the tropics and the connection to the Hadley and Walker circulations. J. Geophys. Res. Atmos., 119, 1322-1339, https://doi.org/10.1002/2013JD020742.

Showman, A. P., and L. M. Polvani, 2011: Equatorial superrotation on tidally locked exoplanets. Astrophys. J., 738, 71, https:// doi.org/10.1088/0004-637X/738/1/71.

Simmons, A., J. Wallace, and G. Branstator, 1983: Barotropic wave propagation and instability, and atmospheric teleconnection patterns. J. Atmos. Sci., 40, 1363-1392, https://doi.org/10.1175/ 1520-0469(1983)040<1363:BWPAIA > 2.0.CO;2.

Swanson, K., 2000: Stationary wave accumulation and the generation of low-frequency variability on zonally varying flows. J. Atmos. Sci., 57, 2262-2280, https://doi.org/10.1175/15200469(2000)057<2262:SWAATG >2.0.CO;2.

Ting, M., and I. M. Held, 1990: The stationary wave response to a tropical SST anomaly in an idealized GCM. J. Atmos. Sci., 47, 2546-2566, https://doi.org/10.1175/1520-0469(1990)047<2546: TSWRTA $>2.0 . \mathrm{CO} ; 2$.

Trenberth, K. E., 1976: Spatial and temporal variations of the Southern Oscillation. Quart. J. Roy. Meteor. Soc., 102, 639653, https://doi.org/10.1002/qj.49710243310.

van der Wiel, K., A. J. Matthews, D. P. Stevens, and M. M. Joshi, 2015: A dynamical framework for the origin of the diagonal South Pacific and South Atlantic convergence zones. Quart. J. Roy. Meteor. Soc., 141, 1997-2010, https://doi.org/10.1002/qj.2508.
, M. M. Joshi, and D. P. Stevens, 2016: Why the South Pacific convergence zone is diagonal. Climate Dyn., 46, 16831698, https://doi.org/10.1007/s00382-015-2668-0.

Wallace, J. M., and D. S. Gutzler, 1981: Teleconnections in the geopotential height field during the Northern Hemisphere winter. Mon. Wea. Rev., 109, 784-812, https://doi.org/10.1175/ 1520-0493(1981)109<0784:TITGHF $>2.0 . \mathrm{CO} ; 2$.

Wang, L., and W. Chen, 2014: An intensity index for the East Asian winter monsoon. J. Climate, 27, 2361-2374, https://doi.org/ 10.1175/JCLI-D-13-00086.1.

Wheeler, M. C., and H. H. Hendon, 2004: An all-season real-time multivariate MJO index: Development of an index for monitoring and prediction. Mon. Wea. Rev., 132, 1917-1932, https:// doi.org/10.1175/1520-0493(2004)132<1917:AARMMI>2. $0 . \mathrm{CO} ; 2$.

Widlansky, M. J., P. J. Webster, and C. D. Hoyos, 2011: On the location and orientation of the South Pacific convergence zone. Climate Dyn., 36, 561-578, https://doi.org/10.1007/s00382-0100871-6.

Zurita-Gotor, P., 2019a: The role of the divergent circulation for large-scale eddy momentum transport in the tropics. Part I: Observations. J. Atmos. Sci., 76, 1125-1144, https://doi.org/ 10.1175/JAS-D-18-0297.1.

_ 2019b: The role of the divergent circulation for large-scale eddy momentum transport in the tropics. Part II: Dynamical determinants of the momentum flux. J. Atmos. Sci., 76, 11451161, https://doi.org/10.1175/JAS-D-18-0304.1.

_ 2020: The impact of divergence tilt and meridional flow for crossequatorial eddy momentum transport in Gill-like settings. J. Atmos. Sci., 77, 1933-1953, https://doi.org/10.1175/JAS-D-19-0158.1.

— ability of the Hadley and Ferrel cells. J. Climate, 31, 47574773, https://doi.org/10.1175/JCLI-D-17-0752.1. 